\title{
Bott periodicity for the topological classification of gapped states of matter with reflection symmetry
}

\author{
Luka Trifunovic and Piet Brouwer \\ Dahlem Center for Complex Quantum Systems and Physics Department, Freie Universität Berlin, \\ Arnimallee 14, 14195 Berlin, Germany
}

(Received 19 July 2017; revised manuscript received 14 September 2017; published 2 November 2017)

\begin{abstract}
Using a dimensional reduction scheme based on scattering theory, we show that the classification tables for topological insulators and superconductors with reflection symmetry can be organized in two period-2 and four period-8 cycles, similar to the Bott periodicity found for topological insulators and superconductors without spatial symmetries. With the help of the dimensional reduction scheme, the classification in arbitrary dimensions $d \geqslant 1$ can be obtained from the classification in one dimension, for which we present a derivation based on relative homotopy groups and exact sequences to classify one-dimensional insulators and superconductors with reflection symmetry. The resulting classification is fully consistent with a comprehensive classification obtained recently by Shiozaki and Sato [Phys. Rev. B 90, 165114 (2014)]. The use of a scattering-matrix-inspired method allows us to address the second descendant $\mathbb{Z}_{2}$ phase, for which the topological nontrivial phase was previously reported to be vulnerable to perturbations that break translation symmetry.
\end{abstract}

DOI: 10.1103/PhysRevB.96.195109

\section{INTRODUCTION}

The nonspatial symmetries (time-reversal symmetry, particle-hole symmetry, chiral symmetry, and their combinations) can be organized in two "complex" and eight "real" Altland-Zirnbauer symmetry classes [1]. For each of these classes, the classification of the topological insulating (noninteracting) phases of matter was recently obtained [2-7]. The classification has a cyclic dependence on the dimensionality, with a period-2 sequence for the complex classes and a period-8 sequence for the real classes. This cyclic structure is known as "Bott periodicity" or "Bott clock" and is firmly embedded in the mathematics of algebraic topology [8] and, in particular, the eight "real" classes in K-theory $[9,10]$.

An appealing physical construction that reproduces the period-2 and period-8 cyclic structure was suggested recently by Fulga and coworkers [11]. Fulga et al. show that the reflection matrix $r_{d}$ of a gapped half-infinite system in $d$ dimensions with Hamiltonian $H_{d}$ can be naturally interpreted as the Hamiltonian $H_{d-1}$ of a gapped system in $d-1$ dimensions, but with a different symmetry, which precisely follows the period- 2 and period- 8 Bott clocks of the complex and real Altland-Zirnbauer classes, respectively. Since topological classification reflection matrix $r_{d}$ essentially amounts to a classification of the boundary of the $d$-dimensional Hamiltonian $H_{d}$, the assumption of bulk-boundary correspondence-a topologically nontrivial bulk is accompanied with gapless boundary states in a unique way - then immediately gives the Bott periodicity for noninteracting gapped phases of matter.

The concept of topological phases has been extended to include (noninteracting) topological phases that are protected by a crystalline symmetry, in addition to the nonspatial symmetries of the Altland-Zirnbauer classification. The additional spatial symmetries give rise to gapless states at the boundary [11-21], provided the boundary is invariant under the symmetry operation. Although the topological crystalline phases rely on spatial symmetries, many topological phases associated with crystalline symmetries are robust to disorder that preserves these symmetries on the average [22-27]. Although no protected gapless boundary states exist for noninvariant boundaries, other boundary-related consequences of a nontrivial topology may persist, such as polarization changes $[20,28,29]$ or lower-dimensional states at the intersection of multiple boundaries [30-35].

Because of the large number of possible spatial symmetries, especially in higher dimensions, the task of classifying crystalline topological phases is a formidable one. Although a comprehensive classification similar to that of the nonspatial symmetries is still lacking, considerable progress has been made. On the one hand, this includes complete classifications for all crystalline symmetries, but restricted to a single AltlandZirnbauer class in two or three dimensions [36-47]. On the other hand, there are classifications for all Altland-Zirnbauer classes and all spatial dimensions, but for a restricted set of crystalline symmetry operations, such as inversion symmetry [48] or reflection symmetry [49-51]. The latter approach was found to yield period- 2 and period- 8 dependencies on the dimension $d$ known from the classification without crystalline symmetries [48,49], including, in some cases, cyclic structures reminiscent of the "Bott clock" [50,51].

Reflection symmetry is one of most often considered crystalline symmetries, and reflection-symmetric materials were the first experimental realizations of crystalline topological insulators [52-54]. A complete classification of reflectionsymmetric topological insulators and superconductors was reported by Chiu et al., employing the method of minimal Dirac Hamiltonians [49]. Morimoto and Furusaki [50], using an approach based on Clifford algebras [3], showed that the topological classes with reflection symmetry can be organized in period- 2 and period- 8 cyclic structures, although for some sequences the cycles involve increasing the dimension $d$ in steps of two, rather than in unit steps, as in the case of the standard Bott periodicity. Shiozaki and Sato generalized these results to all order- 2 unitary and antiunitary crystalline symmetries [51] and corrected some entries in the classification table obtained previously $[49,50]$.

In this paper, we show that the reflection-matrix-based dimensional reduction scheme of Fulga et al. can also be applied to reflection-symmetric topological insulators. Using the scheme of Fulga et al. naturally leads us to consider a "chiral reflection" operation, such that once the chiral 
reflection-symmetric gapped Hamiltonians are included, all symmetry combinations can be grouped in period- 2 and period-8 cyclic sequences, for which the dimension $d$ is increased in unit steps. Our results are in complete agreement with the classification obtained by Shiozaki and Sato [51], who used the Hamiltonian dimensional reduction scheme of Teo and Kane [55] to obtain relations between the corresponding K-groups.

Whereas the reflection-matrix-based dimensional reduction scheme allows one to obtain the classification for arbitrary dimension from the classification at $d=0$ in the absence of spatial symmetries [11], in the presence of reflection symmetry this procedure ends already for dimension $d=1$, since a one-dimensional system has no "boundary" that is mapped onto itself by reflection. To make this article self-contained and to provide an alternative to the existing classification schemes [49-51], we here present a classification of one-dimensional reflection-symmetric topological insulators based on relative homotopy groups and exact sequences, following the approach taken by Turner et al. in their classification of inversion-symmetric topological insulators [56]. In combination with the reflection-matrix-based reduction scheme, the $d=1$ classification gives a complete classification for reflection-symmetric topological insulators in all dimensions $d \geqslant 1$. An additional advantage of the approach of Turner et al. is that it gives explicit expressions for topological invariants and for the generators of the topological classes (many examples are given in Appendix C).

Our approach allows us to address an issue related to stability of the topological phase of the second descendant of $\mathbb{Z}_{2}$ in the classes where reflection symmetry anticommutes with nonspatial symmetries. Chiu et al. and Morimoto and Furusaki argued that the topological $\mathbb{Z}_{2}$ index cannot be defined in these cases and that an eventual topologically nontrivial phase is always weak, i.e., it is instable to perturbations that break the lattice translation symmetry $[49,50]$. While Shiozaki and Sato left open the possibility of a "subtle instability" to translation-symmetry-breaking perturbations, they insisted that the topological invariant is a strong one [51]. Having the explicit form of the topological invariant at our disposal, we can confirm that it is invariant under a redefinition of the unit cell. Moreover, since our reflection-matrix-based approach effectively classifies the boundary of the insulator, we can show explicitly that a nonzero topological invariant implies the existence of a topologically protected boundary state. We find no signs of the instability reported in Refs. [49,50].

This article is organized as follows: In Sec. II, we review the constraints that reflection symmetry poses on the Hamiltonian $H_{d}$ of a gapped system in $d$ dimensions. In Sec. III, we review the reflection-matrix-based method of dimensional reduction originally proposed by Fulga et al. [11] and we show how the method can be generalized to reflection-symmetric topological insulators. The topological classification of onedimensional topological insulators with reflection symmetry using the method of relative homotopy groups and exact sequences is given in Sec. IV. We discuss the controversial second-descendant $\mathbb{Z}_{2}$ phase in Sec. V. We conclude with a brief summary in Sec. VI. Four appendices contain details of the dimensionless reduction scheme, an extension of the $d=1$ classification to higher dimensions (i.e., without the assumption of bulk-boundary correspondence, which underlies the reflection-matrix-based dimensional reduction scheme), explicit examples for topological invariants of onedimensional reflection-symmetric topological insulators, and supporting details for the second-descendant $\mathbb{Z}_{2}$ phase.

\section{SYMMETRIES}

We consider a local Hamiltonian $H_{d}(\mathbf{k})$ in $d$ dimensions, with $\mathbf{k}=\left(k_{1}, k_{2}, \ldots, k_{d}\right)$. For definiteness we take the reflection plane to be perpendicular to the $d$ th unit vector, so that reflection maps the wave vector $\mathbf{k}$ to $R \mathbf{k}=$ $\left(k_{1}, k_{2}, \ldots, k_{d-1},-k_{d}\right)$. We choose the unit cell such that it is mapped to itself under reflection. Reflection nevertheless (in general) affects the basis states in the unit cell, so that for the Hamiltonian $H_{d}(\mathbf{k})$ reflection symmetry takes the form

$$
H_{d}(\mathbf{k})=U_{\mathcal{R}}^{\dagger} H_{d}(R \mathbf{k}) U_{\mathcal{R}},
$$

with $U_{\mathcal{R}}$ being a k-independent unitary matrix (the $k$ independence of $U_{\mathcal{R}}$ is ensured by the choice of the unit cell) [57]. We require $U_{\mathcal{R}}^{2}=1$ to fix the phase freedom in the definition of $U_{\mathcal{R}}$.

The reflection symmetry exists possibly in combination with time-reversal $(\mathcal{T})$, particle-hole $(\mathcal{P})$, and/or chiral $(\mathcal{C})$ symmetries. These symmetries take the form

$$
\begin{gathered}
H_{d}(\mathbf{k})=U_{\mathcal{T}}^{\dagger} H_{d}(-\mathbf{k})^{*} U_{\mathcal{T}}, \\
H_{d}(\mathbf{k})=-U_{\mathcal{P}}^{\dagger} H_{d}(-\mathbf{k})^{*} U_{\mathcal{P}}, \\
H_{d}(\mathbf{k})=-U_{\mathcal{C}}^{\dagger} H_{d}(\mathbf{k}) U_{\mathcal{C}},
\end{gathered}
$$

where $U_{\mathcal{T}}, U_{\mathcal{P}}$, and $U_{\mathcal{C}}$ are $\mathbf{k}$-independent unitary matrices. If time-reversal symmetry and particle-hole symmetry are both present, $U_{\mathcal{C}}=U_{\mathcal{P}} U_{\mathcal{T}}^{*}$. Further, the unitary matrices $U_{\mathcal{T}}, U_{\mathcal{P}}$, and $U_{\mathcal{C}}$ satisfy $U_{\mathcal{T}} U_{\mathcal{T}}^{*}=\mathcal{T}^{2}, U_{\mathcal{P}} U_{\mathcal{P}}^{*}=\mathcal{P}^{2}, U_{\mathcal{C}}^{2}=1$, and $U_{\mathcal{P}} U_{\mathcal{T}}^{*}=\mathcal{T}^{2} \mathcal{P}^{2} U_{\mathcal{T}} U_{\mathcal{P}}^{*}$. [The condition $U_{\mathcal{C}}^{2}=1$ is not fundamental, since multiplication of $U_{\mathcal{C}}$ with a phase factor results in the same chiral symmetry relation (4). We will use this condition to fix signs in intermediate expressions for the general derivation of the Bott clock from scattering theory, but not in the discussion of examples for specific symmetry classes.]

The three nonspatial symmetry operations $\mathcal{T}, \mathcal{P}$, and $\mathcal{C}$ define the ten Altland-Zirnbauer classes [1]. The two complex classes have no symmetries linking $H$ to $H^{*}$; the remaining eight real classes have time-reversal symmetry, particle-hole symmetry, or both. Following common practice in the field, we use Cartan labels to refer to the corresponding symmetry classes; see Table I.

How the presence of a reflection symmetry affects the topological classification depends on whether the reflection operation $\mathcal{R}$ commutes or anticommutes with the nonspatial symmetry operations $\mathcal{T}, \mathcal{P}$, and $\mathcal{C}$. (The condition $U_{\mathcal{R}}^{2}=1$ ensures that the reflection operation $\mathcal{R}$ has well-defined algebraic relations with $\mathcal{T}, \mathcal{P}$, and $\mathcal{C}$.) Following Ref. [49], to distinguish the various cases, we use the symbol $\mathcal{R}$ to denote the presence of reflection symmetry in the absence of any spatial symmetries; $\mathcal{R}_{\sigma_{\mathcal{T}}}, \mathcal{R}_{\sigma_{\mathcal{P}}}$, or $\mathcal{R}_{\sigma_{\mathcal{C}}}$ to denote a reflection symmetry that commutes $(\sigma=+)$ or anticommutes $(\sigma=-)$ 
TABLE I. The ten Altland-Zirnbauer classes are defined according to the presence or absence of time-reversal $(\mathcal{T})$, particle-hole symmetry $(\mathcal{P})$, and chiral symmetry $(\mathcal{C})$. A nonzero entry indicates the square of the antiunitary symmetry operations $\mathcal{T}$ or $\mathcal{P}$.

\begin{tabular}{lcrrrrr}
\hline \hline Class & Cartan & $\mathcal{T}$ & $\mathcal{P}$ & $\mathcal{C}$ & $\pi_{0}$ & $\pi_{1}$ \\
\hline $\mathcal{C}_{0}$ & A & 0 & 0 & 0 & $\mathbb{Z}$ & 0 \\
$\mathcal{C}_{1}$ & AIII & 0 & 0 & 1 & 0 & $\mathbb{Z}$ \\
\hline $\mathcal{R}_{0}$ & AI & 1 & 0 & 0 & $\mathbb{Z}$ & $\mathbb{Z}_{2}$ \\
$\mathcal{R}_{1}$ & BDI & 1 & 1 & 1 & $\mathbb{Z}_{2}$ & $\mathbb{Z}_{2}$ \\
$\mathcal{R}_{2}$ & D & 0 & 1 & 0 & $\mathbb{Z}_{2}$ & 0 \\
$\mathcal{R}_{3}$ & DIII & -1 & 1 & 1 & 0 & $2 \mathbb{Z}$ \\
$\mathcal{R}_{4}$ & AII & -1 & 0 & 0 & $2 \mathbb{Z}$ & 0 \\
$\mathcal{R}_{5}$ & CII & -1 & -1 & 1 & 0 & 0 \\
$\mathcal{R}_{6}$ & C & 0 & -1 & 0 & 0 & 0 \\
$\mathcal{R}_{7}$ & CI & 1 & -1 & 1 & 0 & $\mathbb{Z}$ \\
\hline \hline
\end{tabular}

with the nonspatial symmetry operation $\mathcal{T}, \mathcal{P}$, or $\mathcal{C}$ if only one nonspatial symmetry is present; and $\mathcal{R}_{\sigma_{\mathcal{T}} \sigma_{\mathcal{P}}}$ for a reflection symmetry that commutes or anticommutes with time-reversal symmetry and particle-hole symmetry if all three nonspatial symmetries are present. (If $\mathcal{R}$ neither commutes nor anticommutes with the fundamental nonspatial symmetries, the Hamiltonian $H_{d}(\mathbf{k})$ can be brought into block-diagonal form, such that there are well-defined commutation or anticommutation relations between $\mathcal{R}$ and $\mathcal{T}, \mathcal{P}$, and $\mathcal{C}$ for each of the blocks.) The commutation or anticommutation relations between $\mathcal{R}$ and $\mathcal{T}, \mathcal{P}$, or $\mathcal{C}$ imply the algebraic relations $U_{\mathcal{R}} U_{\mathcal{T}}=\sigma_{\mathcal{T}} U_{\mathcal{T}} U_{\mathcal{R}}^{*}$, $U_{\mathcal{R}} U_{\mathcal{P}}=\sigma_{\mathcal{P}} U_{\mathcal{T}} U_{\mathcal{R}}^{*}$, and $U_{\mathcal{R}} U_{\mathcal{C}}=\sigma_{\mathcal{C}} U_{\mathcal{T}} U_{\mathcal{C}}$ between the unitary matrices implementing these operations.

\section{DIMENSIONAL REDUCTION}

We now describe how one can construct a dimensional reduction scheme consistent with Bott periodicity using reflection matrices. We first review how this method works in the absence of reflection symmetry, as discussed by Fulga et al. [11], and then show how to include the presence of reflection symmetry.

\section{A. Reflection-matrix-based method}

The key step in the method of Ref. [11] is the construction a $(d-1)$-dimensional gapped Hamiltonian $H_{d-1}$ for each $d$ dimensional gapped Hamiltonian $H_{d}$. The Hamiltonians $H_{d}$ and $H_{d-1}$ have different symmetries, but the same (strong) topological invariants. Fulga et al. show how the Hamiltonian $H_{d-1}$ can be constructed from the reflection matrix $r_{d}$ if a gapped system with Hamitonian $H_{d}$ is attached to an ideal lead with a $(d-1)$-dimensional cross section.

Since the reflection matrix depends on the properties of the surface of the $d$-dimensional insulator, this dimensional reduction method assumes that the boundary properties can be used to classify the bulk; i.e., it assumes a bulk-boundary correspondence. This is the case for the standard AltlandZirnbauer classes (without additional symmetries). It is also the case in the presence of a mirror symmetry, provided the surface contains the normal to the mirror plane [49].

To be specific, following Ref. [11] we consider a $d$-dimensional gapped insulator with Hamiltonian

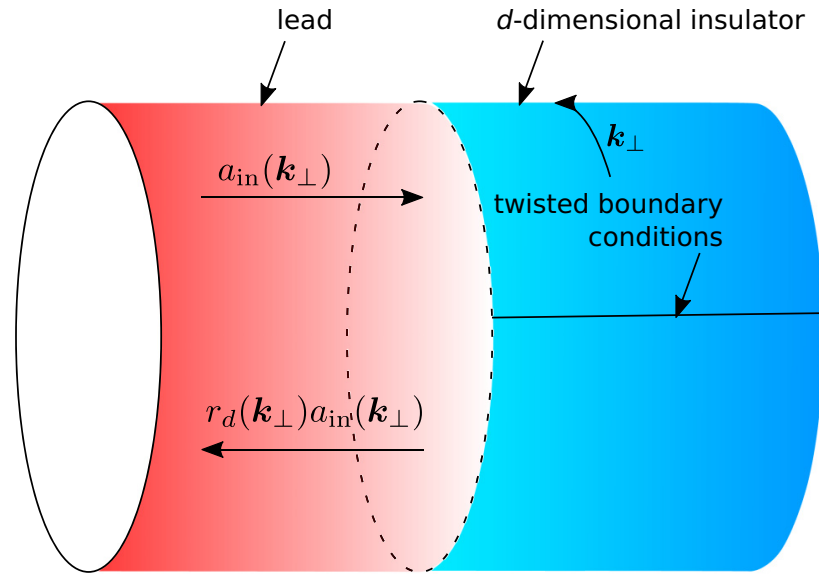

FIG. 1. Schematic picture of a $d$-dimensional gapped insulator occupying the half space (blue) with twisted boundary conditions applied along $(d-1)$-dimension (black line), coupled to an ideal lead (red) with a $(d-1)$-dimensional cross section. The reflection matrix $r_{d}\left(\mathbf{k}_{\perp}\right)$ relates the amplitudes of outgoing and incoming modes in the lead.

$H_{d}(\mathbf{k})=H_{d}\left(k_{1}, \mathbf{k}_{\perp}\right)$, occupying the half space $x_{1}>0$; see Fig. 1 . The half space $x_{1}<0$ consists of an ideal lead with transverse modes labeled by the $(d-1)$-dimensional wave vector $\mathbf{k}_{\perp}$. The amplitudes $a_{\text {out }}\left(\mathbf{k}_{\perp}\right)$ and $a_{\text {in }}\left(\mathbf{k}_{\perp}\right)$ of outgoing and incoming modes are related by the reflection matrix $r_{d}\left(\mathbf{k}_{\perp}\right)$,

$$
a_{\text {out }}\left(\mathbf{k}_{\perp}\right)=r_{d}\left(\mathbf{k}_{\perp}\right) a_{\text {in }}\left(\mathbf{k}_{\perp}\right) .
$$

Since $H_{d}$ is gapped, $r\left(\mathbf{k}_{\perp}\right)$ is unitary. Time-reversal symmetry, particle-hole symmetry, or chiral symmetry pose additional constraints on $r_{d}\left(\mathbf{k}_{\perp}\right)$. These follow from the action of these symmetries on the amplitudes $a_{\text {in }}$ and $a_{\text {out }}$,

$$
\begin{aligned}
\mathcal{T} a_{\text {in }}\left(\mathbf{k}_{\perp}\right) & =Q_{\mathcal{T}} a_{\text {out }}^{*}\left(-\mathbf{k}_{\perp}\right), \\
\mathcal{T} a_{\text {out }}\left(\mathbf{k}_{\perp}\right) & =V_{\mathcal{T}} a_{\text {in }}^{*}\left(-\mathbf{k}_{\perp}\right), \\
\mathcal{P} a_{\text {in }}\left(\mathbf{k}_{\perp}\right) & =V_{\mathcal{P}} a_{\text {in }}^{*}\left(-\mathbf{k}_{\perp}\right), \\
\mathcal{P} a_{\text {out }}\left(\mathbf{k}_{\perp}\right) & =Q_{\mathcal{P}} a_{\text {out }}^{*}\left(-\mathbf{k}_{\perp}\right), \\
\mathcal{C} a_{\text {in }}\left(\mathbf{k}_{\perp}\right) & =Q_{\mathcal{C}} a_{\text {out }}\left(\mathbf{k}_{\perp}\right), \\
\mathcal{C} a_{\text {out }}\left(\mathbf{k}_{\perp}\right) & =V_{\mathcal{C}} a_{\text {in }}\left(\mathbf{k}_{\perp}\right),
\end{aligned}
$$

where $V_{\mathcal{T}}, Q_{\mathcal{T}}, V_{\mathcal{P}}, Q_{\mathcal{P}}, V_{\mathcal{C}}$, and $Q_{\mathcal{C}}$ are $\mathbf{k}_{\perp}$-independent unitary matrices that satisfy $V_{\mathcal{T}} Q_{\mathcal{T}}^{*}=\mathcal{T}^{2}, V_{\mathcal{P}} V_{\mathcal{P}}^{*}=Q_{\mathcal{P}} Q_{\mathcal{P}}^{*}=$ $\mathcal{P}^{2}$, and $Q_{\mathcal{C}} V_{\mathcal{C}}=\mathcal{C}^{2}=1$. Systems with both time-reversal and particle-hole symmetry also have a chiral symmetry, with $Q_{\mathcal{C}}=V_{\mathcal{P}} Q_{\mathcal{T}}^{*}=\mathcal{T}^{2} \mathcal{P}^{2} Q_{\mathcal{T}} Q_{\mathcal{P}}^{*}$ and $V_{\mathcal{C}}=Q_{\mathcal{P}} V_{\mathcal{T}}^{*}=$ $\mathcal{T}^{2} \mathcal{P}^{2} V_{\mathcal{T}} V_{\mathcal{P}}^{*}$. For the reflection matrix $r_{d}\left(\mathbf{k}_{\perp}\right)$, one then finds that the presence of time-reversal symmetry, particle-hole symmetry, and/or chiral symmetry leads to the constraints

$$
\begin{gathered}
r\left(\mathbf{k}_{\perp}\right)=Q_{\mathcal{T}}^{\mathrm{T}} r\left(-\mathbf{k}_{\perp}\right)^{\mathrm{T}} V_{\mathcal{T}}^{*}, \\
r\left(\mathbf{k}_{\perp}\right)=Q_{\mathcal{P}}^{\mathrm{T}} r\left(-\mathbf{k}_{\perp}\right)^{*} V_{\mathcal{P}}^{*}, \\
r\left(\mathbf{k}_{\perp}\right)=Q_{\mathcal{C}}^{\dagger} r\left(\mathbf{k}_{\perp}\right)^{\dagger} V_{\mathcal{C}} .
\end{gathered}
$$


The effective Hamiltonian $H_{d-1}$ is constructed out of $r\left(\mathbf{k}_{\perp}\right)$ in different ways, depending on the presence or absence of chiral symmetry. With chiral symmetry, one sets

$$
H_{d-1}(\mathbf{k}) \equiv Q_{\mathcal{C}} r(\mathbf{k})
$$

using Eq. (11) to verify that $H_{d-1}$ is indeed Hermitian. (Recall that $V_{\mathcal{C}}=Q_{\mathcal{C}}^{\dagger}$ since $Q_{\mathcal{C}} V_{\mathcal{C}}=\mathcal{C}^{2}=1$.) Without chiral symmetry, one defines $H_{d-1}$ as

$$
H_{d-1}(\mathbf{k})=\left(\begin{array}{cc}
0 & r(\mathbf{k}) \\
r^{\dagger}(\mathbf{k}) & 0
\end{array}\right),
$$

which is manifestly Hermitian and satisfies a chiral symmetry with $U_{\mathcal{C}}=\sigma_{3}$.

Bulk-boundary correspondence implies that the bulk, which is described by the Hamiltonian $H_{d}(\mathbf{k})$, and the boundary, which determines the reflection matrix $r_{d}\left(\mathbf{k}_{\perp}\right)$, have the same topological classification. Since $r_{d}\left(\mathbf{k}_{\perp}\right)$ is one-to-one correspondence with the Hamiltonian $H_{d-1}\left(\mathbf{k}_{\perp}\right)$, this implies that $H_{d}$ and $H_{d-1}$ have the same topological classification (provided bulk-boundary correspondence applies). Inspecting the symmetries of the sequence of Hamiltonians that results upon stepwise lowering the dimension $d$, one recovers two periodic sequences of Hamiltonians with the same topological classification. The appearance of a period- 2 sequence for the complex classes

$$
\mathrm{A} \stackrel{d-1}{\longrightarrow} \mathrm{AIII} \stackrel{d-1}{\longrightarrow} \mathrm{A}
$$

follows immediately from the alternating presence and absence of chiral symmetry in the sequence of Hamiltonians $H_{d}$ constructed above. To establish the period- 8 sequence, one needs to inspect how the dimensional reduction affects the symmetries of $H$ if time-reversal symmetry and/or particle-hole symmetry are present. If $H_{d}$ has both time-reversal and particle-hole symmetry, $H_{d-1}$ is given by Eq. (12). From Eqs. (9), (10), and (12), one derives that time-reversal symmetry and particle-hole symmetry of the reflection matrix $r_{d}(\mathbf{k})$ yield identical symmetry constraints for the Hamiltonian $H_{d-1}(\mathbf{k})$,

$$
H_{d-1}(\mathbf{k})=\mathcal{T}^{2} \mathcal{P}^{2} V_{\mathcal{P}}^{\mathrm{T}} H_{d-1}^{*}(-\mathbf{k}) V_{\mathcal{P}}^{*},
$$

which has the form of a particle-hole symmetry if $\mathcal{T}^{2} \mathcal{P}^{2}=-1$, and of a time-reversal symmetry otherwise. In both cases, the symmetry operation squares to $V_{\mathcal{P}} V_{\mathcal{P}}^{*}=\mathcal{P}^{2}$. If $H_{d}$ has time-reversal symmetry but no particle-hole symmetry, one verifies that $H_{d-1}$ satisfies

$$
\begin{aligned}
H_{d-1}(\mathbf{k}) & =U^{\dagger} H_{d-1}(-\mathbf{k})^{*} U, \\
& =-\left(\sigma_{3} U\right)^{\dagger} H_{d-1}(-\mathbf{k})^{*}\left(\sigma_{3} U\right),
\end{aligned}
$$

with

$$
U=\left(\begin{array}{cc}
0 & V_{\mathcal{T}}^{*} \\
Q_{\mathcal{T}}^{*} & 0
\end{array}\right),
$$

which has the form of a time-reversal symmetry squaring to $\mathcal{T}^{2}$ and a particle-hole symmetry squaring to $-\mathcal{T}^{2}$, whereas if $H_{d}$ has particle-hole symmetry but no time-reversal symmetry, $H_{d-1}$ satisfies the symmetry constraints

$$
H_{d-1}(\mathbf{k})=\mathcal{P}^{2} U^{\dagger} H_{d-1}(-\mathbf{k})^{*} U,
$$

$$
=-\mathcal{P}^{2}\left(\sigma_{3} U\right)^{\dagger} H_{d-1}(-\mathbf{k})^{*}\left(\sigma_{3} U\right),
$$

with

$$
U=\left(\begin{array}{cc}
Q_{\mathcal{P}}^{*} & 0 \\
0 & V_{\mathcal{P}}^{*}
\end{array}\right),
$$

which has the form of a time-reversal symmetry squaring to $\mathcal{P}^{2}$ and a particle-hole symmetry squaring to $\mathcal{P}^{2}$. Combining everything, one arrives at the sequence of symmetry classes

$$
\begin{aligned}
& \mathrm{CI} \stackrel{d-1}{\longrightarrow} \mathrm{C} \stackrel{d-1}{\longrightarrow} \mathrm{CII} \stackrel{d-1}{\longrightarrow} \mathrm{AII} \stackrel{d-1}{\longrightarrow} \mathrm{DIII} \\
& \stackrel{d-1}{\longrightarrow} \mathrm{D} \stackrel{d-1}{\longrightarrow} \mathrm{BDI} \stackrel{d-1}{\longrightarrow} \mathrm{AI} \stackrel{d-1}{\longrightarrow} \mathrm{CI},
\end{aligned}
$$

which is the well-known period-8 Bott periodicity known from the classification of topological insulators and superconductors [2-7].

\section{B. Reflection symmetry}

The dimensional reduction based on the calculation of reflection matrices can also be applied in the presence of a reflection symmetry. As in Sec. II, we take the reflection plane to be perpendicular to the $x_{d}$ axis, so that the reflection operator $\mathcal{R}$ maps the lead-system interface onto itself. As with the nonspatial symmetries, the action of the reflection operation on the amplitudes $a_{\text {in }}$ and $a_{\text {out }}$ of incoming and outgoing states in the leads involves multiplication with $\mathbf{k}_{\perp}$-independent unitary matrices,

$$
\begin{gathered}
\mathcal{R} a_{\text {in }}\left(\mathbf{k}_{\perp}\right)=V_{\mathcal{R}} a_{\text {in }}\left(R \mathbf{k}_{\perp}\right), \\
\mathcal{R} a_{\text {out }}\left(\mathbf{k}_{\perp}\right)=Q_{\mathcal{R}} a_{\text {out }}\left(R \mathbf{k}_{\perp}\right),
\end{gathered}
$$

where $R \mathbf{k}_{\perp}=\left(k_{2}, \ldots, k_{d-1},-k_{d}\right)$ denotes the reflected mode vector. The matrices $V_{\mathcal{R}}$ and $Q_{\mathcal{R}}$ satisfy $V_{\mathcal{R}} Q_{\mathcal{R}}=\mathcal{R}^{2}=1$. The presence of reflection symmetry leads to a constraint on the reflection matrix,

$$
r\left(\mathbf{k}_{\perp}\right)=Q_{\mathcal{R}}^{\dagger} r\left(R \mathbf{k}_{\perp}\right) V_{\mathcal{R}} .
$$

The algebraic relations involving the matrices $Q_{\mathcal{R}}, V_{\mathcal{R}}$ depend on whether the reflection operation $\mathcal{R}$ commutes or anticommutes with the nonspatial symmetry operations $\mathcal{T}, \mathcal{P}$, and $\mathcal{C}, Q_{\mathcal{T}} Q_{\mathcal{R}}^{*}=\sigma_{\mathcal{T}} V_{\mathcal{R}} Q_{\mathcal{T}}, V_{\mathcal{T}} V_{\mathcal{R}}^{*}=\sigma_{\mathcal{T}} Q_{\mathcal{R}} V_{\mathcal{T}}$, $V_{\mathcal{P}} V_{\mathcal{R}}^{*}=\sigma_{\mathcal{P}} V_{\mathcal{R}} V_{\mathcal{P}}, Q_{\mathcal{P}} Q_{\mathcal{R}}^{*}=\sigma_{\mathcal{P}} Q_{\mathcal{R}} Q_{\mathcal{P}}, Q_{\mathcal{C}} Q_{\mathcal{R}}=\sigma_{\mathcal{C}} V_{\mathcal{R}} Q_{\mathcal{C}}$, and $V_{\mathcal{C}} V_{\mathcal{R}}=\sigma_{\mathcal{C}} Q_{\mathcal{R}} V_{\mathcal{C}}$.

To see how the presence of reflection symmetry affects the dimensional reduction, we first consider the complex classes A and AIII. Starting from a Hamiltonian $H_{d}$ in symmetry class A with reflection symmetry $\mathcal{R}$, we construct a Hamiltonian $H_{d-1}$ in class AIII as described above and find that reflection symmetry imposes the constraint

$$
H_{d-1}\left(\mathbf{k}_{\perp}\right)=U_{\mathcal{R}}^{\dagger} H_{d-1}\left(R \mathbf{k}_{\perp}\right) U_{\mathcal{R}},
$$

on $H_{d-1}$, with

$$
U_{\mathcal{R}}=\left(\begin{array}{cc}
Q_{\mathcal{R}} & 0 \\
0 & V_{\mathcal{R}}
\end{array}\right) .
$$

Since $U_{\mathcal{R}}$ commutes with $\sigma_{3}$, we conclude that dimensional reduction maps the class $\mathrm{A}^{\mathcal{R}}$ to $\mathrm{AIII}^{\mathcal{R}_{+}}$. A similar procedure can be applied to a Hamiltonian $H_{d}$ in class AIII with reflection symmetry $\mathcal{R}_{\sigma_{\mathcal{C}}}$, with $\sigma_{\mathcal{C}}= \pm$. In this case, one finds that 
dimensional reduction leads to a Hamiltonian $H_{d-1}$ in class A with the additional constraint

$$
H_{d-1}\left(\mathbf{k}_{\perp}\right)=\sigma_{\mathcal{C}} V_{\mathcal{R}}^{\dagger} H_{d-1}\left(R \mathbf{k}_{\perp}\right) V_{\mathcal{R}} .
$$

This constraint has the form of a reflection symmetry if $\sigma_{\mathcal{C}}=1$, i.e., if $\mathcal{R}$ commutes with $\mathcal{C}$, but not if $\sigma_{\mathcal{C}}=-1$, i.e., if $\mathcal{R}$ anticommutes with $\mathcal{C}$. Instead, if $\sigma_{\mathcal{C}}=-1$ the constraint (24) represents the product of a reflection symmetry and a chiral symmetry. We denote such a combined symmetry operation with the symbol $\mathcal{C} R$. To complete the analysis, we consider a Hamiltonian $H_{d}$ in class A with the $\mathcal{C} R$ symmetry constraint,

$$
H_{d}(\mathbf{k})=-U_{\mathcal{C} R}^{\dagger} H_{d}(R \mathbf{k}) U_{\mathcal{C} R},
$$

where $R \mathbf{k}=\left(k_{1}, k_{2}, \ldots, k_{d-1},-k_{d}\right)$. On the level of the reflection matrix $r_{d}\left(\mathbf{k}_{\perp}\right)$, the $\mathcal{C} R$ symmetry is implemented as

$$
r_{d}\left(\mathbf{k}_{\perp}\right)=Q_{\mathcal{C} R}^{\dagger} r_{d}\left(R \mathbf{k}_{\perp}\right)^{\dagger} V_{\mathcal{C} R},
$$

where $Q_{\mathcal{C} R} V_{\mathcal{C} R}=1$ and $R \mathbf{k}_{\perp}=\left(k_{2}, \ldots, k_{d-1},-k_{d}\right)$. By performing the dimensional reduction scheme to this Hamiltonian $H_{d}$, one immediately finds that $H_{d-1}$ satisfies the constraint

$$
H_{d-1}\left(\mathbf{k}_{\perp}\right)=U_{\mathcal{R}}^{\dagger} H_{d-1}\left(R \mathbf{k}_{\perp}\right) U_{\mathcal{R}}
$$

with

$$
U_{\mathcal{R}}=\left(\begin{array}{cc}
0 & Q_{\mathcal{C} R} \\
V_{\mathcal{C} R} & 0
\end{array}\right) .
$$

Since $U_{\mathcal{R}}$ anticommutes with $\sigma_{3}$, the constraint Eq. (27) has the form of a reflection symmetry that anticommutes with the chiral symmetry $\mathcal{C}$. Combining everything, we conclude that once the symmetry operation $\mathcal{C} R$ is added, the dimensional reduction scheme for the complex classes with reflection symmetry leads to two period-2 sequences,

$$
\begin{gathered}
\mathrm{A}^{\mathcal{R}} \stackrel{d-1}{\longrightarrow} \mathrm{AIII}^{\mathcal{R}_{+}} \stackrel{d-1}{\longrightarrow} \mathrm{A}^{\mathcal{R}}, \\
\mathrm{A}^{\mathcal{C R}} \stackrel{d-1}{\longrightarrow} \mathrm{AIII}^{\mathcal{R}_{-}} \stackrel{d-1}{\longrightarrow} \mathrm{A}^{\mathcal{C R}} .
\end{gathered}
$$

The symmetry operation $\mathcal{C} R$ naturally appears in the dimensional reduction scheme for the real classes as well. As with the standard reflection symmetry, we have to distinguish between the cases $\mathcal{C} R_{\sigma_{\mathcal{T}, \mathcal{P}}}$ that the $\mathcal{C} R$ symmetry operation commutes $(\sigma=+)$ or anticommutes $(\sigma=-)$ with the time-reversal or particle-hole symmetry operations, if one of these symmetries is present. (If both symmetries are present, there is no need to consider $\mathcal{C} R$ as a separate symmetry operation.) The relations (25) and (26) also apply to the general case. If chiral symmetry is present, one has $V_{\mathcal{C} R}=V_{\mathcal{C}} V_{\mathcal{R}}$ and $Q_{\mathcal{C} R}=Q_{\mathcal{C}} Q_{\mathcal{R}}$. One further has the algebraic relations $Q_{\mathcal{T}} Q_{\mathcal{C} R}^{*}=\sigma_{\mathcal{T}} V_{\mathcal{C} R} Q_{\mathcal{T}}, V_{\mathcal{T}} V_{\mathcal{C} R}^{*}=\sigma_{\mathcal{T}} Q_{\mathcal{C} R} V_{\mathcal{T}}$, $V_{\mathcal{P}} V_{\mathcal{C} R}^{*}=\sigma_{\mathcal{P}} V_{\mathcal{C} R} V_{\mathcal{P}}, Q_{\mathcal{P}} Q_{\mathcal{C} R}^{*}=\sigma_{\mathcal{P}} Q_{\mathcal{C} R} Q_{\mathcal{P}}$. Proceeding as above, one verifies that the dimensional reduction scheme then leads to four period-8 sequences,

$$
\begin{aligned}
& \mathrm{CI}^{\mathcal{R}_{++}} \stackrel{d-1}{\longrightarrow} \mathrm{C}^{\mathcal{R}_{+}} \stackrel{d-1}{\longrightarrow} \mathrm{CII}^{\mathcal{R}_{++}} \stackrel{d-1}{\longrightarrow} \mathrm{AII}^{\mathcal{R}_{+}} \\
& \stackrel{d-1}{\longrightarrow} \mathrm{DIII}^{\mathcal{R}_{++}} \stackrel{d-1}{\longrightarrow} \mathrm{D}^{\mathcal{R}_{+}} \stackrel{d-1}{\longrightarrow} \mathrm{BDI}^{\mathcal{R}_{++}} \\
& \stackrel{d-1}{\longrightarrow} \mathrm{AI}^{\mathcal{R}_{+}} \stackrel{d-1}{\longrightarrow} \mathrm{CI}^{\mathcal{R}_{++}},
\end{aligned}
$$

$$
\begin{aligned}
\mathrm{CI}^{\mathcal{R}_{--}} & \stackrel{d-1}{\longrightarrow} \mathrm{C}^{\mathcal{R}_{-}} \stackrel{d-1}{\longrightarrow} \mathrm{CII}^{\mathcal{R}_{--}} \stackrel{d-1}{\longrightarrow} \mathrm{AII}^{\mathcal{R}_{-}} \\
& \stackrel{d-1}{\longrightarrow} \mathrm{DIII}^{\mathcal{R}_{--}} \stackrel{d-1}{\longrightarrow} \mathrm{D}^{\mathcal{R}_{-}} \stackrel{d-1}{\longrightarrow} \mathrm{BDI}^{\mathcal{R}_{--}} \\
& \stackrel{d-1}{\longrightarrow} \mathrm{AI}^{\mathcal{R}_{-}} \stackrel{d-1}{\longrightarrow} \mathrm{CI}^{\mathcal{R}_{--}}, \\
\mathrm{CI}^{\mathcal{R}_{-+}} & \stackrel{d-1}{\longrightarrow} \mathrm{C}^{\mathcal{C} \mathcal{R}_{+}} \stackrel{d-1}{\longrightarrow} \mathrm{CII}^{\mathcal{R}_{+-}} \stackrel{d-1}{\longrightarrow} \mathrm{AII}^{\mathcal{C} \mathcal{R}_{-}} \\
& \stackrel{d-1}{\longrightarrow} \mathrm{DIII}^{\mathcal{R}_{-+}} \stackrel{d-1}{\longrightarrow} \mathrm{D}^{\mathcal{C} \mathcal{R}_{+}} \stackrel{d-1}{\longrightarrow} \mathrm{BDI}^{\mathcal{R}_{+-}} \\
& \stackrel{d-1}{\longrightarrow} \mathrm{AI}^{\mathcal{C} \mathcal{R}_{-}} \stackrel{d-1}{\longrightarrow} \mathrm{CI}^{\mathcal{R}_{-+}}, \\
\mathrm{CI}^{\mathcal{R}_{+-}} & \stackrel{d-1}{\longrightarrow} \mathrm{C}^{\mathcal{C} \mathcal{R}_{-}} \stackrel{d-1}{\longrightarrow} \mathrm{CII}^{\mathcal{R}_{-+}} \stackrel{d-1}{\longrightarrow} \mathrm{AII}^{\mathcal{C R}_{+}} \\
& \stackrel{d-1}{\longrightarrow} \mathrm{DIII}^{\mathcal{R}_{+-}} \stackrel{d-1}{\longrightarrow} \mathrm{D}^{\mathcal{C} \mathcal{R}_{-}} \stackrel{d-1}{\longrightarrow} \mathrm{BDI}^{\mathcal{R}_{-+}} \\
& \stackrel{d-1}{\longrightarrow} \mathrm{AI}^{\mathcal{C} \mathcal{R}_{+}} \stackrel{d-1}{\longrightarrow} \mathrm{CI}^{\mathcal{R}_{+-}} .
\end{aligned}
$$

Details of the derivation are given in Appendix A. The above sequences were first derived by Morimoto and Furusaki but "skipping" the classes containing $\mathcal{C} \mathcal{R}$ symmetry [50]. Shiozaki and Sato obtained the relations between K-groups that give all the sequences derived here as a special case [51].

\section{TOPOLOGICAL CLASSIFICATION WITH REFLECTION SYMMETRY}

Having established the dimensional reduction scheme, it is sufficient to consider the case $d=1$ in order to completely classify gapped Hamiltonians with reflection symmetry. (The dimensional reduction scheme cannot be used down to $d=$ 0 because there can be no reflection-invariant lead-system interface in one dimension.) Various methods have been used in the literature to accomplish this task [49-51], as discussed in the introduction and in the review article, Ref. [58].

To make this paper self-contained, we here include a systematic classification of reflection-symmetric gapped Hamiltonians for $d=1$. We have chosen to use a different method than used in Refs. [49-51], which makes use of concepts from algebraic topology, using relative homotopy groups and exact sequences. This method was used by Turner et al. for their classification of topological insulators with inversion symmetry [56]. In Appendix B, we discuss how this classification method can be directly applied to reflectionsymmetric Hamiltonians in dimensions $d>1$, without the use of the reflection-matrix-based dimensional reduction scheme (and, hence, without the implicit assumption of bulk-boundary correspondence).

The construction of a topological classification for the Hamiltonians $H_{d}$ requires a mathematical formalism that endows the space of Hamiltonians with a group structure. The theory of vector bundles and the "Grothendieck group" provides such a formal structure, essentially using the diagonal addition of Hamiltonians as the group addition operation. Both concepts are reviewed in a language accessible to physicists, e.g., in Ref. [59] and in the appendix of Ref. [56]. We here employ a more informal language, noting that a formally correct formulation requires an interpretation of our statements in the framework of the vector bundles and the Grothendieck group. As in the previous section, we use the Cartan labels 


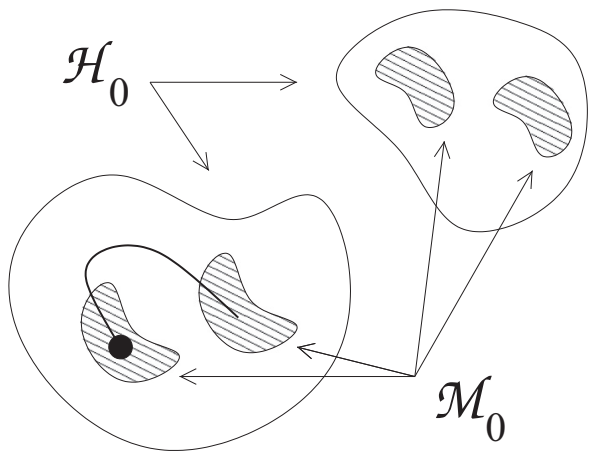

FIG. 2. Schematic illustration of the spaces $\mathcal{H}_{0}$ and $\mathcal{M}_{0}$. The solid dot indicates the trivial element. The thick curve shows a path in $\mathcal{H}_{0}$ that starts at the trivial element and ends in $\mathcal{M}_{0}$. Equivalence classes of such paths form the relative homotopy group $\pi_{1}\left(\mathcal{H}_{0}, \mathcal{M}_{0}\right)$.

to denote the space of Hermitian matrices $H$ with a gapped spectrum for the two complex and eight real Altland-Zirnbauer symmetry classes; see Table I.

In one dimension, we are interested in periodic, continuous functions $H(k)=H(k+2 \pi)$, with $H(k)$ being a gapped Hamiltonian, where the antiunitary symmetry operations $\mathcal{T}$ and $\mathcal{P}$ as well as the reflection operations $\mathcal{R}$ and $\mathcal{C} R$ relate $H(k)$ and $H(-k)$. It is then sufficient to consider the Hamiltonian $H(k)$ on the interval $0 \leqslant k \leqslant \pi$ only. For generic $0<k<\pi$, only symmetries that relate $H(k)$ to itself play a role. These symmetries confine $H(k)$ for $0<k<\pi$ to one of the classifying spaces of Table I. We use the symbol $\mathcal{H}_{0}$ to denote this space. The momenta $k=0$ and $k=\pi$ are mapped to themselves under $k \rightarrow-k$, so that $H(0)$ and $H(\pi)$ satisfy additional symmetries. We use $\mathcal{M}_{0}$ to denote the classifying space of Hamiltonians that also satisfy these additional symmetry constraints. Figure 2 schematically illustrates the spaces $\mathcal{H}_{0}$ and $\mathcal{M}_{0}$.

In general, a Hamiltonian $H(k)$ can be block decomposed as $H(k)=H(0) \oplus H^{\prime}(k)$, where $H^{\prime}(0)$ is topologically trivial. The $k$-independent Hamiltonian $H(0)$ has topological indices characteristic of the zero-dimensional case. These indices become weak indices of one-dimensional Hamiltonian $H(k)$. The classification of the Hamiltonians $H^{\prime}(k)$ gives the strong topological indices.

In view of these considerations, our goal is to classify functions $H^{\prime}(k)$ on the interval $0 \leqslant k \leqslant \pi$, such that $H^{\prime}(k)$ is gapped, $H^{\prime}(0)$ is trivial, $H^{\prime}(\pi) \in \mathcal{M}_{0}$, and $H(k) \in \mathcal{H}_{0}$ otherwise. The space of equivalence classes (defined with respect to continuous deformations) of such functions $H^{\prime}(k)$ is known as the relative homotopy group $\pi_{1}\left(\mathcal{H}_{0}, \mathcal{M}_{0}\right)[56,60]$. The group $\pi_{1}\left(\mathcal{H}_{0}, \mathcal{M}_{0}\right)$ gives the topological classification of gapped Hamiltonians with the desired symmetries. A function $H^{\prime}(k)$ with these constraints can be interpreted as a continuous path in $\mathcal{H}_{0}$, starting at the trivial point and ending somewhere in $\mathcal{M}_{0}$; see Fig. 2.

The relative homotopy group can be calculated from the zeroth and first homotopy groups of $\mathcal{H}_{0}$ and $\mathcal{M}_{0}$, where we recall that the zeroth homotopy groups $\pi_{0}(\mathcal{X})$ labels the connected components of a topological space $\mathcal{X}$, whereas the first homotopy group $\pi_{1}(\mathcal{X})$ contains equivalence classes of "closed loops" in $\mathcal{X}$ that begin and end at the trivial reference
TABLE II. The complete classification for the complex AltlandZirnbauer classes with reflection symmetry.

\begin{tabular}{lcccccc}
\hline \hline Class & $\mathcal{H}_{0}^{i}$ & $\mathcal{M}_{0}^{i}$ & $d=1$ & $d=2$ & $d=3$ & $d=4$ \\
\hline $\mathrm{A}^{\mathcal{R}}$ & $\mathrm{A}$ & $\mathrm{A}^{2}$ & $\mathbb{Z}$ & 0 & $\mathbb{Z}$ & 0 \\
$\mathrm{AIII}^{\mathcal{R}_{+}}$ & $\mathrm{AIII}$ & $\mathrm{AIII}^{2}$ & 0 & $\mathbb{Z}$ & 0 & $\mathbb{Z}$ \\
\hline $\mathrm{A}^{\mathcal{C} \mathcal{R}}$ & A & AIII & 0 & $\mathbb{Z}^{2}$ & 0 & $\mathbb{Z}^{2}$ \\
$\mathrm{AIII}^{\mathcal{R}_{-}}$ & AIII & A & $\mathbb{Z}^{2}$ & 0 & $\mathbb{Z}^{2}$ & 0 \\
\hline \hline
\end{tabular}

point. This calculation makes use of an "exact sequence" of mappings [56]

$$
\begin{aligned}
\pi_{1}\left(\mathcal{M}_{0}\right) & \stackrel{i_{1}}{\rightarrow} \pi_{1}\left(\mathcal{H}_{0}\right) \stackrel{j_{1}}{\hookrightarrow} \pi_{1}\left(\mathcal{H}_{0}, \mathcal{M}_{0}\right) \\
& \stackrel{\delta}{\rightarrow} \pi_{0}\left(\mathcal{M}_{0}\right) \stackrel{i_{0}}{\hookrightarrow} \pi_{0}\left(\mathcal{H}_{0}\right),
\end{aligned}
$$

where a sequence of mappings is called "exact" if the image of each mapping is the kernel of the subsequent one. In the sequence (35), the maps $i_{1}, j_{1}$, and $i_{0}$ are inclusion maps where the same object is interpreted as an element of a larger space. The map $\delta$ is the "boundary map", mapping an equivalence class of "paths" $H(k)$ in $\pi_{1}\left(\mathcal{H}_{0}, \mathcal{M}_{0}\right)$ to the connected component of their endpoint $H(\pi)$ in $\mathcal{M}_{0}$. Since the groups $\pi_{1}\left(\mathcal{H}_{0}\right)$ and $\pi_{0}\left(\mathcal{M}_{0}\right)$ as well as the image of $i_{1}$ and the kernel of $i_{0}$ are known, the relative homotopy group $\pi_{1}\left(\mathcal{H}_{0}, \mathcal{M}_{0}\right)$ and its structure follow immediately from the exactness of the sequence (35). Similarly, generators for $\pi_{1}\left(\mathcal{H}_{0}, \mathcal{M}_{0}\right)$ can be constructed by application of the inclusion map $j_{1}$ and a suitable inverse of the boundary map $\delta$. Table I lists the groups $\pi_{0}$ and $\pi_{1}$ for the classifying spaces $\mathcal{C}_{n}$ and $\mathcal{R}_{n}$.

To classify one-dimensional gapped Hamiltonians with reflection symmetry, the spaces $\mathcal{H}_{0}$ and $\mathcal{M}_{0}$ are identified for each symmetry class (see Tables II and III) for the two period-2 complex sequences and for the four period-8 real sequences, respectively. The relative homotopy group $\pi_{1}\left(\mathcal{H}_{0}, \mathcal{M}_{0}\right)$, which classifies the gapped Hamiltonians with reflection or $\mathcal{C} \mathcal{R}$ symmetry, is then calculated from the exact sequence (35). The results of this classification are shown in Tables II and III. In addition to the classification for $d=1$, the table also lists the results for $d=2,3$, and 4 , following the Bott clock structure outlined in the previous section. The assignment of the spaces $\mathcal{H}_{0}$ and $\mathcal{M}_{0}$ for the different symmetry classes and the details on the resolution of the exact sequence in the nontrivial cases is discussed in detail in Appendix C.

\section{THE SECOND DESCENDANT $\mathbb{Z}_{2}$ PHASE}

Chiu et al. [49] and Morimoto and Furusaki [50] argued that the class $\mathrm{CII}^{\mathcal{R}_{--}}$of reflection-symmetric topological superconductors in two dimensions $(d=2)$ has gapless boundary states that not protected against perturbations that lift the discrete translation symmetry of the underlying lattice. On the other hand, Shiozaki and Sato point out that this class has a well-defined strong index, although they nevertheless allow for a "subtle instability" of the topologically nontrivial state [51].

The dimensional reduction scheme links class $\mathrm{CII}^{\mathcal{R}_{--}}$with $d=2$ to class $\mathrm{AII}^{\mathcal{R}_{-}}$in one dimension; i.e., the reflection 
TABLE III. The complete classification for the real AltlandZirnbauer classes with reflection symmetry.

\begin{tabular}{|c|c|c|c|c|c|c|}
\hline Class & $\mathcal{H}_{0}^{i}$ & $\mathcal{M}_{0}^{i}$ & $d=1$ & $d=2$ & $d=3$ & $d=4$ \\
\hline $\mathrm{AI}^{\mathcal{R}_{+}}$ & AI & $\mathrm{AI}^{2}$ & $\mathbb{Z}$ & 0 & 0 & 0 \\
\hline $\mathrm{BDI}^{\mathcal{R}_{++}}$ & BDI & $\mathrm{BDI}^{2}$ & $\mathbb{Z}_{2}$ & $\mathbb{Z}$ & 0 & 0 \\
\hline $\mathrm{D}^{\mathcal{R}_{+}}$ & $\mathrm{D}$ & $\mathrm{D}^{2}$ & $\mathbb{Z}_{2}$ & $\mathbb{Z}_{2}$ & $\mathbb{Z}$ & 0 \\
\hline DIII $^{\mathcal{R}_{++}}$ & DIII & $\mathrm{DIII}^{2}$ & 0 & $\mathbb{Z}_{2}$ & $\mathbb{Z}_{2}$ & $\mathbb{Z}$ \\
\hline $\mathrm{AII}^{\mathcal{R}_{+}}$ & AII & $\mathrm{AII}^{2}$ & $2 \mathbb{Z}$ & 0 & $\mathbb{Z}_{2}$ & $\mathbb{Z}_{2}$ \\
\hline $\mathrm{CII}^{\mathcal{R}_{++}}$ & CII & $\mathrm{CII}^{2}$ & 0 & $2 \mathbb{Z}$ & 0 & $\mathbb{Z}_{2}$ \\
\hline $\mathrm{C}^{\mathcal{R}_{+}}$ & $\mathrm{C}$ & $\mathrm{C}^{2}$ & 0 & 0 & $2 \mathbb{Z}$ & 0 \\
\hline $\mathrm{CI}^{\mathcal{R}_{++}}$ & CI & $\mathrm{CI}^{2}$ & 0 & 0 & 0 & $2 \mathbb{Z}$ \\
\hline $\mathrm{AI}^{\mathcal{R}_{-}}$ & AII & $\mathrm{A}$ & 0 & 0 & $2 \mathbb{Z}$ & 0 \\
\hline $\mathrm{BDI}^{\mathcal{R}_{--}}$ & CII & AIII & 0 & 0 & 0 & $2 \mathbb{Z}$ \\
\hline $\mathrm{D}^{\mathcal{R}_{-}}$ & $\mathrm{C}$ & A & $\mathbb{Z}$ & 0 & 0 & 0 \\
\hline DIII $^{\mathcal{R}_{--}}$ & CI & AIII & $\mathbb{Z}_{2}$ & $\mathbb{Z}$ & 0 & 0 \\
\hline $\mathrm{AII}^{\mathcal{R}_{-}}$ & AI & A & $\mathbb{Z}_{2}$ & $\mathbb{Z}_{2}$ & $\mathbb{Z}$ & 0 \\
\hline $\mathrm{CII}^{\mathcal{R}_{--}}$ & BDI & AIII & 0 & $\mathbb{Z}_{2}$ & $\mathbb{Z}_{2}$ & $\mathbb{Z}$ \\
\hline $\mathrm{C}^{\mathcal{R}_{-}}$ & $\mathrm{D}$ & A & $2 \mathbb{Z}$ & 0 & $\mathbb{Z}_{2}$ & $\mathbb{Z}_{2}$ \\
\hline $\mathrm{CI}^{\mathcal{R}_{--}}$ & DIII & AIII & 0 & $2 \mathbb{Z}$ & 0 & $\mathbb{Z}_{2}$ \\
\hline $\mathrm{AI}^{\mathcal{C} \mathcal{R}_{-}}$ & $\mathrm{C}$ & CI & 0 & 0 & 0 & $2 \mathbb{Z}^{2}$ \\
\hline $\mathrm{BDI}^{\mathcal{R}_{+-}}$ & CI & $\mathrm{AI}$ & $\mathbb{Z}^{2}$ & 0 & 0 & 0 \\
\hline $\mathrm{D}^{\mathcal{C R} \mathcal{R}_{+}}$ & AI & BDI & $\mathbb{Z}_{2}^{2}$ & $\mathbb{Z}^{2}$ & 0 & 0 \\
\hline DIII $^{\mathcal{R}_{-+}}$ & BDI & $\mathrm{D}$ & $\mathbb{Z}_{2}^{2}$ & $\mathbb{Z}_{2}^{2}$ & $\mathbb{Z}^{2}$ & 0 \\
\hline $\mathrm{AII}^{\mathcal{C} \mathcal{R}_{-}}$ & $\mathrm{D}$ & DIII & 0 & $\mathbb{Z}_{2}^{\frac{2}{2}}$ & $\mathbb{Z}_{2}^{2}$ & $\mathbb{Z}^{2}$ \\
\hline $\mathrm{CII}^{\mathcal{R}_{+-}}$ & DIII & AII & $2 \mathbb{Z}^{2}$ & 0 & $\mathbb{Z}_{2}^{2}$ & $\mathbb{Z}_{2}^{2}$ \\
\hline $\mathrm{C}^{\mathcal{C} \mathcal{R}_{+}}$ & AII & CII & 0 & $2 \mathbb{Z}^{2}$ & 0 & $\mathbb{Z}_{2}^{2}$ \\
\hline$\underline{\mathrm{CI}^{\mathcal{R}_{-+}}}$ & $\mathrm{CII}$ & $\mathrm{C}$ & 0 & 0 & $2 \mathbb{Z}^{2}$ & 0 \\
\hline $\mathrm{AI}^{\mathcal{C} \mathcal{R}_{+}}$ & $\mathrm{D}$ & BDI & 0 & $2 \mathbb{Z}$ & 0 & $\mathbb{Z}$ \\
\hline $\mathrm{BDI}^{\mathcal{R}_{-+}}$ & DIII & $\mathrm{D}$ & $\mathbb{Z}$ & 0 & $2 \mathbb{Z}$ & 0 \\
\hline $\mathrm{D}^{\mathcal{C R} \mathcal{R}_{-}}$ & AII & DIII & 0 & $\mathbb{Z}$ & 0 & $2 \mathbb{Z}$ \\
\hline $\mathrm{DIII}^{\mathcal{R}_{+-}}$ & CII & AII & $2 \mathbb{Z}$ & 0 & $\mathbb{Z}$ & 0 \\
\hline $\mathrm{AII}^{\mathcal{C} \mathcal{R}_{+}}$ & $\mathrm{C}$ & CII & 0 & $2 \mathbb{Z}$ & 0 & $\mathbb{Z}$ \\
\hline $\mathrm{CII}^{\mathcal{R}_{-+}}$ & CI & $\mathrm{C}$ & $\mathbb{Z}$ & 0 & $2 \mathbb{Z}$ & 0 \\
\hline $\mathrm{C}^{\mathcal{C R} \mathcal{R}_{-}}$ & AI & CI & 0 & $\mathbb{Z}$ & 0 & $2 \mathbb{Z}$ \\
\hline $\mathrm{CI}^{\mathcal{R}_{+-}}$ & BDI & AI & $2 \mathbb{Z}$ & 0 & $\mathbb{Z}$ & 0 \\
\hline
\end{tabular}

matrix $r_{2}\left(k_{\perp}\right)$ of a two-dimensional Hamiltonian $H_{2}(\mathbf{k})$ in class $\mathrm{CII}^{\mathcal{R}_{--}}$is a one-dimensional object with symmetries characteristic of class $\mathrm{AII}^{\mathcal{R}_{-}}$. In this section, we show that the definition of the $\mathbb{Z}_{2}$ topological invariant for class $\mathrm{AII}^{\mathcal{R}_{-}}$ is robust to the addition of perturbations that break the discrete translation symmetry, consistent with the observation of Shiozaki and Sato that there is a well-defined topological index [51]. We then use our scattering approach to show that a nontrivial value of the invariant implies the existence of gapless states at the boundary of the two-dimensional system. As explained in Appendix E, we believe the fact that Refs. [49] and [50] observe a gap opening for edge states is because the perturbation considered there includes a long-range hopping term with a hopping amplitude decaying inversely proportional to distance, which does not result in a continuous Bloch Hamiltonian $H(\mathbf{k})$ as a function of $\mathbf{k}$.

The class $\mathrm{AII}^{\mathcal{R}_{-}}$has time-reversal symmetry with $\mathcal{T}^{2}=$ -1 . Combining the reflection and time-reversal symmetries, we arrive at

$$
(\mathcal{R T}) H(k)(\mathcal{R T})=H(k)
$$

with $(\mathcal{R} \mathcal{T})^{2}=1$ since $\mathcal{R}$ and $\mathcal{T}$ anticommute. Without loss of generality, we may represent $\mathcal{R} \mathcal{T}$ by complex conjugation $K$ and $\mathcal{R}$ by $\sigma_{2}$, so that $H(k)$ is a real symmetric matrix with the additional condition $H(k)=\sigma_{2} H(-k) \sigma_{2}$. We conclude that $\mathcal{H}_{0}$ is the class AI, whereas at the reflection symmetric points $k=0, \pi, H$ is of the form

$$
H=\left(\begin{array}{cc}
a & b \\
-b & a
\end{array}\right)
$$

with $a(b)$ real symmetric (antisymmetric). Such a structure forms a two-dimensional representation of the complex numbers, so that we find that $\mathcal{M}_{0}$ is the space of gapped Hamiltonians in class A. Following the general procedure of Sec. IV, we write $H(k)=H(0) \oplus H^{\prime}(k)$, where $H^{\prime}(0)$ is topologically trivial. This gives the exact sequence

$$
0 \stackrel{i_{1}}{\hookrightarrow} \mathbb{Z}_{2} \stackrel{j_{1}}{\hookrightarrow} \mathbb{Z}_{2} \stackrel{\delta}{\rightarrow} \mathbb{Z} \stackrel{i_{0}}{\hookrightarrow} \mathbb{Z},
$$

with $\pi_{1}\left(\mathcal{H}_{0}, \mathcal{M}_{0}\right)=\mathbb{Z}_{2}$. The topological structure is inherited from the left part of the exact sequence. The index can be calculated as the standard invariant classifying loops of real symmetric matrices [61],

$$
W^{\prime}=\prod_{k=0, \pi} \operatorname{Pf} w(k),
$$

with (recall that the time-reversal operation $\mathcal{T}$ maps $k$ to $-k$ )

$$
w_{a b}(k)=-w_{b a}(k)=-i\left\langle u(-k)_{a}|\mathcal{T}| u_{b}(k)\right\rangle,
$$

where $\left|u_{a}(k)\right\rangle$ is the real Bloch wave functions of occupied states chosen to be continuous functions of $k$ for $-\pi \leqslant k \leqslant \pi$, but it is not necessary with the periodicity property that $\left|u_{a}(-\pi)\right\rangle$ be equal to $\left|u_{a}(\pi)\right\rangle$. At the reflection-symmetric points $k=0$, $\pi$, the matrix $w(k)$ is orthogonal and skew symmetric, so that the Pfaffian can only take values \pm 1 .

The Hamiltonian

$$
H^{(-1)}(k)=\left(\begin{array}{cc}
\cos k \tau_{3}+\sin k \tau_{1} & 0 \\
0 & \cos k \tau_{3}-\sin k \tau_{1}
\end{array}\right)
$$

is a generator with $W^{\prime}=-1$. This can be seen by noting that the eigenvectors of the negative-energy states are

$$
\left|u_{1}(k)\right\rangle=\left(\begin{array}{c}
\sin (k / 2) \\
-\cos (k / 2) \\
0 \\
0
\end{array}\right),\left|u_{2}(k)\right\rangle=\left(\begin{array}{c}
0 \\
0 \\
\sin (k / 2) \\
\cos (k / 2)
\end{array}\right),
$$

from which it follows that

$$
w(k)=\left(\begin{array}{cc}
0 & \cos k \\
-\cos k & 0
\end{array}\right)
$$

and $W^{\prime}=-1$. We note that the value of the topological invariant (39) depends on the choice of the unit cell if the number of occupied bands is an odd multiple of two. For example, for the four-band model (41), the two choices of the unit cell shown in Fig. 3(a), corresponding to the two possible positions of the reflection plane, give two different values of the topological invariant, although they both represent the same physical system [62]. This ambiguity does not influence the presence of protected gapless modes of reflection-symmetric boundaries of two-dimensional systems, since these depend on the product of topological invariants 
(a)
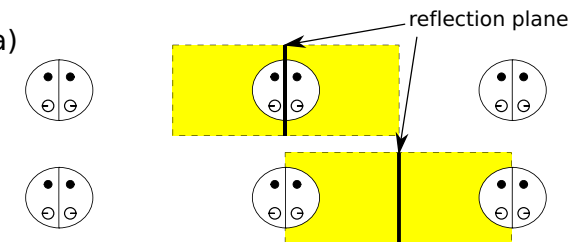

(b)
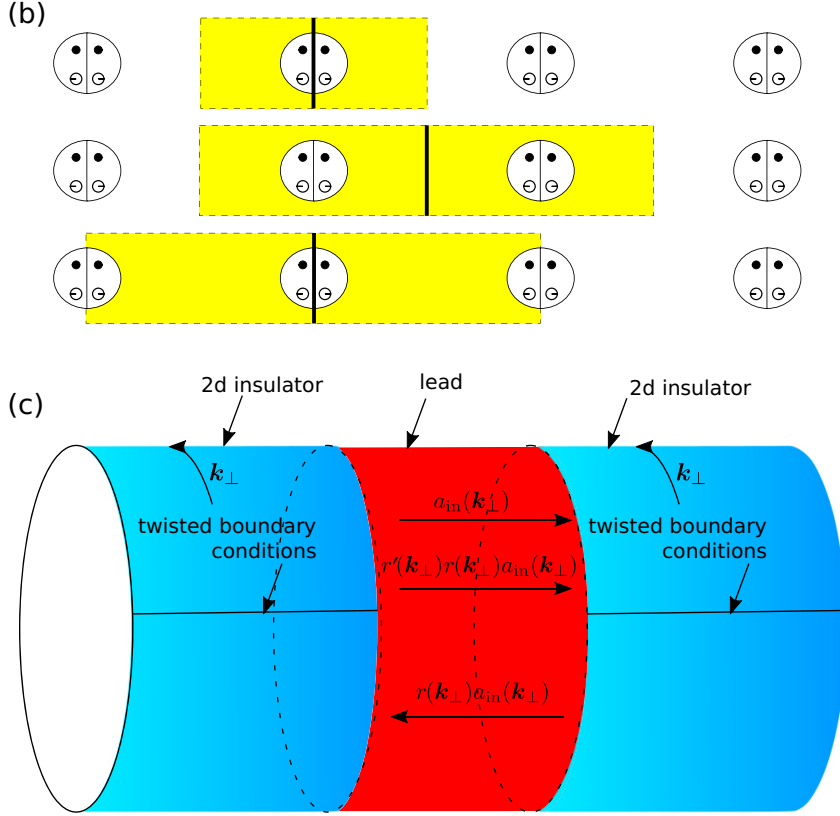

FIG. 3. (a) Schematic illustration of the two nonequivalent positions for the reflection plane (solid vertical lines) and the corresponding choice of the unit cell (yellow box). (b) Two nonequivalent ways of doubling unit cell. The first row depicts the unit cell before the doubling; the second row shows the doubled unit cell where the reflection axis has been moved by half of the lattice period; the third row gives the choice of the unit cell that is consistent with original reflection axis. (c) Schematic illustration of a two-dimensional insulator, attached to an ideal lead of finite length. The insulator has reflection matrix $r$, and the lead is terminated with reflection matrix $r^{\prime}$. This setup is used to obtain the boundary state from the scattering approach.

of the form (41) on the two sides of the boundary; see the discussion at the end of this section. If the number of the occupied bands is an even multiple of two, the topological invariant (41) is independent of the choice of the unit cell and the above-mentioned ambiguity does not arise.

At this point, we would like to stress again that in the classification procedure weak topological numbers, i.e., topological numbers that are not robust against translationsymmetry breaking, are associated with $H(0)$, not with $H^{\prime}(k)$. Hence, the invariant $W^{\prime}$ should be a strong invariant. To see this explicitly, we now show that the nontrivial topological index for the Hamiltonian $H^{(-1)}(k)$ of Eq. (41) survives the redefinition of the unit cell that comes with the breaking of translation symmetry. Indeed, out of Eq. (41) one can construct an eight-band Hamiltonian $H_{2}^{(-1)}(k)$ as

$$
H_{2}^{(-1)}(k)=e^{-i \mu_{2} k / 2}\left[H^{(-1)}(k) \oplus H^{(-1)}(k+\pi)\right] e^{i \mu_{2} k / 2},
$$

where the Pauli matrix $\mu_{2}$ acts in the space consisting of the two original unit cells that form the new unit cell. One verifies that the unit cell for $H_{2}^{(-1)}(k)$ is twice that of the original Hamiltonian $H^{(-1)}(k)$, i.e., that $H_{2}^{(-1)}(k)=H_{2}^{(-1)}(k+\pi)$, and that $H_{2}^{(-1)}(k)$ has the same symmetries as $H^{(-1)}(k)$, defined by $\mathcal{R}=\mu_{3} \sigma_{2}$ and $\mathcal{R} \mathcal{T}=K$. Upon doubling the unit cell, we changed the position of the reflection plane, such that the new unit cell maps onto itself under reflection-otherwise the reflection operator would become $k$ dependent; see Fig. 3(b). A direct calculation gives that the invariant (39) is the same for both $H^{(-1)}(k)$ and $H_{2}^{(-1)}(k)$. [If instead we would have kept the position of the reflection plane the same and correspondingly adapted the choice of the unit cell as shown in the third line of Fig. 3(b), the doubling procedure would result in topological invariant $-W^{\prime}$ if the number of occupied bands is an odd multiple of two, which still leaves the product of invariants on the two sides of a reflection symmetric sample-boundary and, hence, the presence or absence of protected boundary states, unaffected.] Similarly, one may construct Hamiltonians in which the unit cell size is multiplied by an odd number, with the same properties; see Appendix D. We conclude that the invariant (39) is a true strong invariant.

It remains to show that a nontrivial value of the topological invariant implies the existence of a gapless boundary state. To relate the reflection matrix to boundary states, we consider a two-dimensional topological insulator in class $\mathrm{CII}^{\mathcal{R}_{--}}$and attach an ideal lead to the left; see Fig. 3. As discussed previously, the reflection matrix $r\left(k_{\perp}\right)$ (when multiplied by $Q_{\mathcal{C}}$ ) belongs to symmetry class $\mathrm{AII}^{\mathcal{R}_{-}}$. To model a sample edge, the lead is terminated on its left end by a reflection matrix $r^{\prime}\left(k_{\perp}\right)$ which, again, is in symmetry class AII ${ }^{\mathcal{R}}$ (when multiplied by $Q_{\mathcal{C}}$ ). The condition to have a boundary state at zero energy is

$$
\operatorname{det}\left(\mathbb{1}-r\left(k_{\perp}\right) r^{\prime}\left(k_{\perp}\right)\right)=0 .
$$

If the two-dimensional bulk is in a nontrivial topological class in $\mathrm{CII}^{\mathcal{R}_{--}}$, the two matrices $r\left(k_{\perp}\right)$ and $r^{\prime}\left(k_{\perp}\right)$ are in different topological classes in $\mathrm{AII}^{\mathcal{R}_{-}}$. We now show that this is sufficient to ensure the existence of zero-energy boundary states, i.e., that Eq. (45) has a solution for at least one value of $k_{\perp}$.

We first note that $Q_{\mathcal{C}} r\left(k_{\perp}\right)$ and $r^{\prime}\left(k_{\perp}\right) Q_{\mathcal{C}}$ are Hermitian and unitary, so that all eigenvalues are 1 or -1 , the number of negative eigenvalues being the same for all $k_{\perp}$. Moreover, at $k=0$ and $k=\pi, Q_{\mathcal{C}} r\left(k_{\perp}\right)$ and $r^{\prime}\left(k_{\perp}\right) Q_{\mathcal{C}}$ all eigenvalues are twofold degenerate because of the block structure (37). If the number of positive and negative eigenvalues are not balanced, the product $r\left(k_{\perp}\right) r^{\prime}\left(k_{\perp}\right)$ trivially has a unit eigenvalue for all $k_{\perp}$, i.e., there is a "flat band" of surface states. Hence, we may restrict ourselves to the case that the number of positive and negative eigenvalues are equal. This implies that the dimension of $r$ and $r^{\prime}$ is $4 N$, with $N$ integer, as the eigenvalues of $Q_{\mathcal{C}} r\left(k_{\perp}\right)$ and $r^{\prime}\left(k_{\perp}\right) Q_{\mathcal{C}}$ are twofold degenerate at $k_{\perp}=0, \pi$.

We take real bases $\left\{\left|u_{j}\left(k_{\perp}\right)\right\rangle\right\}$ and $\left\{\left|u_{j}^{\prime}\left(k_{\perp}\right)\right\rangle\right\}$ for the negative-eigenvalue eigenspaces of $Q_{\mathcal{C}} r\left(k_{\perp}\right)$ and $r^{\prime}\left(k_{\perp}\right) Q_{\mathcal{C}}$, respectively, with the constraint that

$$
\left|u_{2 l}(0)\right\rangle=i \mathcal{T}\left|u_{2 l-1}(0)\right\rangle, \quad l=1,2, \ldots, N,
$$


and similar for the primed basis set. This gives $\operatorname{Pf}(w(0))=$ $\operatorname{Pf}\left(w^{\prime}(0)\right)=1$, where $w$ and $w^{\prime}$ are the antisymmetric matrices defined for $Q_{\mathcal{C}} r\left(k_{\perp}\right)$ and $r^{\prime}\left(k_{\perp}\right) Q_{\mathcal{C}}$, respectively; see Eq. (40). We then define the $4 N \times 4 N$ real matrix $O\left(k_{\perp}\right)$ with the first (second) $2 N$ columns given by the vectors $\left|u_{j}\left(k_{\perp}\right)\right\rangle$ $\left(\left\{\left|u_{j}^{\prime}\left(k_{\perp}\right)\right\rangle\right), j=1,2, \ldots, 2 N\right.$. Below we show that either $\operatorname{det} O(0) \operatorname{det} O(\pi)=0$ or

$$
\text { sign } \operatorname{det} O(0) \operatorname{det} O(\pi)=W_{r}^{\prime} W_{r^{\prime}}^{\prime},
$$

where $W_{r}^{\prime}$ and $W_{r^{\prime}}^{\prime}$ are the topological numbers for $Q_{\mathcal{C}} r\left(k_{\perp}\right)$ and $r^{\prime}\left(k_{\perp}\right) Q_{\mathcal{C}}$, respectively. In both cases, it follows that $\operatorname{det} O\left(k_{\perp}\right)$ is zero for at least one value of $k_{\perp}$, i.e., the vectors $\left\{\left|u_{j}\left(k_{\perp}\right)\right\rangle,\left|u_{j}^{\prime}\left(k_{\perp}\right)\right\rangle\right\}$ are linearly dependent and hence do not span the full $4 N$-dimensional space. Since

$$
\begin{aligned}
& Q_{\mathcal{C}} r\left(k_{\perp}\right)=\mathbb{1}-2 \sum_{j=1}^{2 N}\left|u_{j}\left(k_{\perp}\right)\right\rangle\left\langle u_{j}\left(k_{\perp}\right)\right|, \\
& r^{\prime}\left(k_{\perp}\right) Q_{\mathcal{C}}=\mathbb{1}-2 \sum_{j=1}^{2 N}\left|u_{j}^{\prime}\left(k_{\perp}\right)\right\rangle\left\langle v_{j}\left(k_{\perp}\right)\right|,
\end{aligned}
$$

it follows that $r\left(k_{\perp}\right) r^{\prime}\left(k_{\perp}\right)$ has an eigenvalue one and thus Eq. (45) guarantees the existence of a boundary state for that value of $k_{\perp}$.

Equation (47) follows immediately if the negativeeigenvalue subspaces of $r^{\prime}\left(k_{\perp}\right) Q_{\mathcal{C}}$ and $Q_{\mathcal{C}} r\left(k_{\perp}\right)$ each are the same at $k_{\perp}=0$ and $k_{\perp}=\pi$. In that case, the topological numbers $W_{r}^{\prime}$ and $W_{r^{\prime}}^{\prime}$ give the "handedness" of the transformation, linking the basis states at $k_{\perp}=0$ and at $k_{\perp}=\pi$,

$$
W_{r}^{\prime}=\operatorname{sign} \operatorname{det}\left[\left\langle u_{i}(\pi) \mid u_{j}(0)\right\rangle\right]
$$

with a similar equality for $W_{r^{\prime}}^{\prime}$. The determinant $\operatorname{det} O(\pi)$ gives the relative handedness of the two transformations, if the basis sets $\left\{\left|u_{j}\left(k_{\perp}\right)\right\rangle\right\}$ and $\left\{\left|v_{j}\left(k_{\perp}\right)\right\rangle\right\}$ are linearly independent at $k_{\perp}=0, \pi$, so that Eq. (47) follows. (If the basis sets $\left\{\left|u_{j}\left(k_{\perp}\right)\right\rangle\right\}$ and $\left\{\left|v_{j}\left(k_{\perp}\right)\right\rangle\right\}$ are not linearly independent at $k_{\perp}=0, \pi$, one has det $O(0) \operatorname{det} O(\pi)=0$.) In the general case, one can show that Eq. (47) holds by comparing the basis sets $\left\{\left|u_{j}\left(k_{\perp}\right)\right\rangle\right\}$ and $\left\{\left|u_{j}^{\prime}\left(k_{\perp}\right)\right\rangle\right\}$ at $k_{\perp}=\pi$ with two real reference basis sets $\left\{\left|\tilde{u}_{j}(s)\right\rangle\right\}$ and $\left\{\left|\tilde{u}_{j}^{\prime}(s)\right\rangle\right\}, 0 \leqslant s \leqslant \pi$, which are identical to the original sets $\left\{\left|u_{j}\left(k_{\perp}\right)\right\rangle\right\}$ and $\left\{\left|u_{j}^{\prime}\left(k_{\perp}\right)\right\rangle\right\}$ at $k_{\perp}=0$, span the same subspaces as the original sets $\left\{\left|u_{j}\left(k_{\perp}\right)\right\rangle\right\}$ and $\left\{\left|u_{j}^{\prime}\left(k_{\perp}\right)\right\rangle\right\}$ at $k_{\perp}=\pi$, and satisfy the constraint (46) at all values of $0 \leqslant$ $s \leqslant \pi$. (Here the time-reversal operation does not send $s$ to $-s$.) The topological invariant $W_{r}^{\prime}$ can then be calculated as the "handedness" of the transformation between the basis sets $\left\{\left|u_{j}(\pi)\right\rangle\right\}$ and $\left\{\left|\tilde{u}_{j}(\pi)\right\rangle\right\}$,

$$
W_{r}^{\prime}=\operatorname{sign} \operatorname{det}\left[\left\langle u_{i}(\pi) \mid \tilde{u}_{j}(\pi)\right\rangle\right],
$$

with a similar result for the topological invariant $W_{r^{\prime}}^{\prime}$. The desired result now follows from the observation that shifting the reference basis sets from $s=\pi$ to $s=0$ does not change the handedness of the transformation. In Appendix E, we carry out the analysis of the present section, numerically on a concrete example.

\section{SUMMARY}

We have studied the classification of topological insulators and superconductors in the presence of reflection symmetry. We used method based on the reflection matrix to derive the Bott clock which is in agreement with previous works [50,51]. For the sake of completeness, we also obtained the classification using a method based on relative homotopy groups and exact sequences; our results are in full agreement with those of Shizoaki et al. [51] and partial agreement with Refs. [49,50]. We also show that the nontrivial topological phases classified with second descendant $\mathbb{Z}_{2}$ are robust to disorder.

The dimensional reduction scheme based on the reflection matrix is distinguished by being physically intuitive since it relates the topological invariant to transport properties of a system. Additionally it offers a high computational efficiency when studying the effects of disorder since one can consider systems in lower dimension. The dimensional reduction method used in this work is purely algebraic and can be readily extended to other point group symmetries; together with relative exact sequences this method could yield the complete classification of topological phases of matter.

\section{ACKNOWLEDGMENTS}

We thank Akira Furusaki, Cosma Fulga, and Shinsei Ryu for stimulating discussions. We acknowledge support by project A03 of the CRC-TR 183 and by the priority programme SPP 1666 of the German Science Foundation (DFG).

\section{APPENDIX A: DIMENSIONAL REDUCTION WITH REFLECTION SYMMETRY}

Here we give the details of the calculation of four period- 8 sequences for the real classes with reflection symmetry. We need to distinguish three cases separately: (i) $H_{d}$ has both time-reversal and particle-hole symmetry, (ii) $H_{d}$ has timereversal symmetry but no particle-hole symmetry, and (iii) $H_{d}$ has particle-hole symmetry but no time-reversal symmetry.

(i) If $H_{d}$ has both time-reversal and particle-hole symmetry, then $H_{d-1}$ satisfies

$$
\begin{gathered}
H_{d-1}(\mathbf{k})=\mathcal{T}^{2} \mathcal{P}^{2} V_{\mathcal{P}}^{\mathrm{T}} H_{d-1}^{*}(-\mathbf{k}) V_{\mathcal{P}}^{*}, \\
H_{d-1}(\mathbf{k})=\sigma_{\mathcal{C}} V_{\mathcal{R}}^{\dagger} H_{d-1}(R \mathbf{k}) V_{\mathcal{R}} .
\end{gathered}
$$

The first constraint has the form of a particle-hole symmetry if $\mathcal{T}^{2} \mathcal{P}^{2}=-1$, and of a time-reversal symmetry otherwise. The second constraint has the form of reflection symmetry $(\mathcal{C R}$ symmetry) if $\sigma_{\mathcal{C}}=1\left(\sigma_{\mathcal{C}}=-1\right)$, with the algebraic relation to the nonspatial symmetry $\left(\mathcal{T}\right.$ or $\mathcal{P}$ ) given by $\sigma_{\mathcal{P}}$.

(ii) If $H_{d}$ has time-reversal symmetry but no particle-hole symmetry, one has

$$
\begin{array}{r}
H_{d-1}(\mathbf{k})=U^{\dagger} H_{d-1}(-\mathbf{k})^{*} U \\
=-\left(\sigma_{3} U\right)^{\dagger} H_{d-1}(-\mathbf{k})^{*}\left(\sigma_{3} U\right), \\
H_{d-1}(\mathbf{k})=U_{\mathcal{R}}^{\dagger} H_{d-1}(R \mathbf{k}) U_{\mathcal{R}} \\
H_{d-1}(\mathbf{k})=U_{\mathcal{C} \mathcal{R}}^{\dagger} H_{d-1}(R \mathbf{k}) U_{\mathcal{C R}}
\end{array}
$$


with

$$
\begin{aligned}
U & =\left(\begin{array}{cc}
0 & V_{\mathcal{T}}^{*} \\
Q_{\mathcal{T}}^{*} & 0
\end{array}\right), \\
U_{\mathcal{R}} & =\left(\begin{array}{cc}
Q_{\mathcal{R}} & 0 \\
0 & V_{\mathcal{R}}
\end{array}\right), \\
U_{\mathcal{C R}} & =\left(\begin{array}{cc}
0 & Q_{\mathcal{C R}} \\
V_{\mathcal{C R}} & 0
\end{array}\right) .
\end{aligned}
$$

Here the first constraint has form of a time-reversal symmetry squaring to $\mathcal{T}^{2}$ and a particle-hole symmetry squaring to $-\mathcal{T}^{2}$, while the second (third) constraint has form of reflection symmetry with the same (different) algebraic relation to $\mathcal{T}$ and $\mathcal{P}$ given by $\sigma_{\mathcal{T}}\left(\sigma_{\mathcal{T}}\right.$ and $-\sigma_{\mathcal{T}}$, respectively).

(iii) If $H_{d}$ has particle-hole symmetry but no time-reversal symmetry, one has

$$
\begin{gathered}
H_{d-1}(\mathbf{k})=\mathcal{P}^{2} U^{\dagger} H_{d-1}(-\mathbf{k})^{*} U \\
=-\mathcal{P}^{2}\left(\sigma_{3} U\right)^{\dagger} H_{d-1}(-\mathbf{k})^{*}\left(\sigma_{3} U\right), \\
H_{d-1}(\mathbf{k})=U_{\mathcal{R}}^{\dagger} H_{d-1}(R \mathbf{k}) U_{\mathcal{R}} \\
H_{d-1}(\mathbf{k})=U_{\mathcal{C} \mathcal{R}}^{\dagger} H_{d-1}(R \mathbf{k}) U_{\mathcal{C R}}
\end{gathered}
$$

with

$$
\begin{aligned}
U_{\mathcal{R}} & =\left(\begin{array}{cc}
Q_{\mathcal{C} R} & 0 \\
0 & V_{\mathcal{C} R}
\end{array}\right), \\
U_{\mathcal{R}} & =\left(\begin{array}{cc}
Q_{\mathcal{R}} & 0 \\
0 & V_{\mathcal{R}}
\end{array}\right), \\
U_{\mathcal{C R}} & =\left(\begin{array}{cc}
0 & Q_{\mathcal{C R}} \\
V_{\mathcal{C R}} & 0
\end{array}\right) .
\end{aligned}
$$

In this case, the first constraint has form of a time-reversal symmetry squaring to $\mathcal{P}^{2}$ and a particle-hole symmetry squaring to $\mathcal{P}^{2}$, while the second (third) constraint has form of reflection symmetry with the same (different) algebraic relation to $\mathcal{T}$ and $\mathcal{P}$ given by $\sigma_{\mathcal{P}}\left(\sigma_{\mathcal{P}}\right.$ and $-\sigma_{\mathcal{P}}$, respectively).

\section{APPENDIX B: ALTERNATIVE DERIVATION OF BOTT CLOCK}

It is possible to establish the Bott clock structure of the classification tables, Tables II and III, without the use of the reflection-matrix-based dimensional reduction scheme. Here we again consider the generalization of the exact sequence (35) to $d>1$. We first note that the spaces $\mathcal{H}_{0}$ and $\mathcal{M}_{0}$ now contain $(d-1)$-dimensional gapped Hamiltonians $H\left(\mathbf{k}^{\prime}\right)$, where the $(d-1)$-dimensional wave vector $\mathbf{k}^{\prime}=\left(k_{1}, k_{2}, \ldots, k_{d-1}\right)$ contains the directions parallel to the reflection hyperplane. By combining reflection symmetry and time-reversal or particlehole symmetry, the antiunitary symmetries that defined the spaces $\mathcal{H}_{0}$ and $\mathcal{M}_{0}$ now act as involutions linking $H\left(\mathbf{k}^{\prime}\right)$ to $H\left(-\mathbf{k}^{\prime}\right)^{*}$. The space of such functions, with the additional condition that $H\left(\mathbf{k}^{\prime}\right)$ be trivial on the (hyper)planes $k_{\alpha}=0$, $\alpha=1,2, \ldots, d-1$, is denoted $\bar{\Omega}^{d-1} \mathcal{C}_{n}$ and $\bar{\Omega}^{d-1} \mathcal{R}_{n}$, for the complex and real classes, respectively, where, instead of the Cartan labels, we use the symbols $\mathcal{C}_{n}$ and $\mathcal{R}_{n}$ to refer to the Altland-Zirnbauer classes; see Table I. Hence, the proper generalization of the Hamiltonian spaces $\mathcal{H}_{0}$ and $\mathcal{M}_{0}$ to the
TABLE IV. The complete list of the exact sequences for $d=1$ complex class topological insulators with reflection symmetry. The table consists of two subtables, within each subtable, the classifying spaces $\mathcal{H}_{0}$ and $\mathcal{M}_{0}$ run along the Bott clock.

\begin{tabular}{lccc}
\hline \hline Class & $\mathcal{H}_{0}$ & $\mathcal{M}_{0}$ & Exact sequence \\
\hline $\mathrm{A}^{\mathcal{R}}$ & $\mathrm{A}$ & $\mathrm{A}^{2}$ & $0 \stackrel{i_{1}}{\hookrightarrow} 0 \stackrel{j_{1}}{\hookrightarrow} \mathbb{Z} \stackrel{\delta}{\rightarrow} \mathbb{Z}^{2} \stackrel{i_{0}}{\hookrightarrow} \mathbb{Z}$ \\
$\mathrm{AIII}^{\mathcal{R}_{+}}$ & AIII & AIII $^{2}$ & $\mathbb{Z}^{2} \stackrel{i_{1}}{\hookrightarrow} \mathbb{Z} \stackrel{j_{1}}{\hookrightarrow} 0 \stackrel{\delta}{\rightarrow} 0 \stackrel{i_{0}}{\hookrightarrow} 0$ \\
\hline $\mathrm{A}^{\mathcal{C} \mathcal{R}}$ & A & AIII & $\mathbb{Z} \stackrel{i_{1}}{\hookrightarrow} 0 \stackrel{j_{1}}{\hookrightarrow} 0 \stackrel{\delta}{\rightarrow} 0 \stackrel{i_{0}}{\hookrightarrow} 0$ \\
$\mathrm{AIII}^{\mathcal{R}_{-}}$ & AIII & A & $0 \stackrel{i_{1}}{\hookrightarrow} \mathbb{Z} \stackrel{j_{1}}{\hookrightarrow} \mathbb{Z}^{2} \stackrel{\delta}{\rightarrow} \mathbb{Z} \stackrel{i_{0}}{\hookrightarrow} 0$ \\
\hline \hline
\end{tabular}

$d$-dimensional case are the spaces $\bar{\Omega}^{d-1} \mathcal{H}_{0}$ and $\bar{\Omega}^{d-1} \mathcal{M}_{0}$. The corresponding generalization of the exact sequence (35) then reads

$$
\begin{aligned}
\pi_{1}\left(\bar{\Omega}^{d-1} \mathcal{M}_{0}\right) & \stackrel{i_{1}}{\hookrightarrow} \pi_{1}\left(\bar{\Omega}^{d-1} \mathcal{H}_{0}\right) \stackrel{j_{1}}{\hookrightarrow} \pi_{1}\left(\bar{\Omega}^{d-1} \mathcal{H}_{0}, \bar{\Omega}^{d-1} \mathcal{M}_{0}\right) \\
& \stackrel{\delta}{\rightarrow} \pi_{0}\left(\bar{\Omega}^{d-1} \mathcal{M}_{0}\right) \stackrel{i_{0}}{\hookrightarrow} \pi_{0}\left(\bar{\Omega}^{d-1} \mathcal{H}_{0}\right) .
\end{aligned}
$$

A central result in K-theory is that the zeroth and first homotopy groups appearing in the exact sequence (35) satisfy a periodicity rule,

$$
\begin{aligned}
\pi_{m}\left(\bar{\Omega}^{d} \mathcal{C}_{n}\right) & =\pi_{m}\left(\mathcal{C}_{n-d} \bmod 2\right), \\
\pi_{m}\left(\bar{\Omega}^{d} \mathcal{R}_{n}\right) & =\pi_{m}\left(\mathcal{R}_{n-d} \bmod 8\right) .
\end{aligned}
$$

This allows one to directly map the exact sequence for the $d$-dimensional case to the exact sequence for the onedimensional case. Since the assignment of the spaces $\mathcal{H}_{0}$ and $\mathcal{M}_{0}$ follows the Bott clock structure (see Tables IV and V), the periodicty implied by Eq. (B2) immediately extends the Bott clock structure to arbitrary dimensions larger than one.

\section{APPENDIX C: TOPOLOGICAL CLASSIFICATION FOR $d=1$}

In Tables IV and V, we give the Cartan labels for the spaces $\mathcal{H}_{0}$ and $\mathcal{M}_{0}$, as well as the corresponding exact sequences for each of the 36 symmetry classes with reflection and/or $\mathcal{C R}$ symmetry. Below we give details for a few special cases that need additional considerations to resolve the sequence and that were not considered in the main text.

\section{The class AIII ${ }^{\mathcal{R}-}$ in $d=1$}

Following Sec. IV, we write $H(k)=H(0) \oplus H^{\prime}(k)$, where $H^{\prime}(0)$ is topologically trivial, and use the method of Sec. IV to classify the Hamiltonians $H^{\prime}(k)$. Without loss of generality, we may choose the unitary operators encoding chiral and reflection operations as $U_{\mathcal{C}}=\sigma_{3}, U_{\mathcal{R}}=\sigma_{1}$, which ensures that $\mathcal{C}$ and $\mathcal{R}$ anticommute. With this choice, chiral symmetry implies that $H^{\prime}(k)$ has the form [63]

$$
H^{\prime}(k)=\left(\begin{array}{cc}
0 & r^{\prime}(k) \\
r^{\prime}(k)^{\dagger} & 0
\end{array}\right),
$$

whereas reflection symmetry imposes the further constraint $r^{\prime}(k)=r^{\prime}(-k)^{\dagger}$. In particular, the blocks $r^{\prime}(0)$ and $r^{\prime}(\pi)$ at the reflection-symmetric momemta $k=0, \pi$ are Hermitian. The numbers $N^{\prime}(0)$ and $N^{\prime}(\pi)$ of negative eigenvalues of $r^{\prime}(0)$ or $r^{\prime}(\pi)$ determines the number of occupied states at $k=0, \pi$ that 
TABLE V. The complete list of the exact sequences for $d=1$ real class topological insulators and superconductors with reflection symmetry. The table consists of four subtables, and within each subtable, the classifying spaces $\mathcal{H}_{0}$ and $\mathcal{M}_{0}$ run along the Bott clock.

\begin{tabular}{|c|c|c|c|}
\hline Class & $\mathcal{H}_{0}$ & $\mathcal{M}_{0}$ & Exact sequence \\
\hline $\mathrm{AI}^{\mathcal{R}_{+}}$ & AI & $\mathrm{AI}^{2}$ & $\mathbb{Z}_{2}^{2} \stackrel{i_{1}}{\hookrightarrow} \mathbb{Z}_{2} \stackrel{j_{1}}{\hookrightarrow} \mathbb{Z} \stackrel{\delta}{\rightarrow} \mathbb{Z}^{2} \stackrel{i_{0}}{\hookrightarrow} \mathbb{Z}$ \\
\hline $\mathrm{BDI}^{\mathcal{R}_{++}}$ & BDI & $\mathrm{BDI}^{2}$ & $\mathbb{Z}_{2}^{2} \stackrel{i_{1}}{\hookrightarrow} \mathbb{Z}_{2} \stackrel{j_{1}}{\hookrightarrow} \mathbb{Z}_{2} \stackrel{\delta}{\rightarrow} \mathbb{Z}_{2}^{2} \stackrel{i_{0}}{\hookrightarrow} \mathbb{Z}_{2}$ \\
\hline $\mathrm{D}^{\mathcal{R}_{+}}$ & $\mathrm{D}$ & $\mathrm{D}^{2}$ & $0 \stackrel{i_{1}}{\hookrightarrow} 0 \stackrel{j_{1}}{\hookrightarrow} \mathbb{Z}_{2} \stackrel{\delta}{\rightarrow} \mathbb{Z}_{2}^{2} \stackrel{i_{0}}{\hookrightarrow} \mathbb{Z}_{2}$ \\
\hline DIII $^{\mathcal{R}_{++}}$ & DIII & $\mathrm{DIII}^{2}$ & $2 \mathbb{Z}^{2} \stackrel{i_{1}}{\hookrightarrow} 2 \mathbb{Z} \stackrel{j_{1}}{\hookrightarrow} 0 \stackrel{\delta}{\rightarrow} 0 \stackrel{i_{0}}{\hookrightarrow} 0$ \\
\hline $\mathrm{AII}^{\mathcal{R}_{+}}$ & AII & $\mathrm{AII}^{2}$ & $0 \stackrel{i_{1}}{\hookrightarrow} 0 \stackrel{j_{1}}{\hookrightarrow} 2 \mathbb{Z} \stackrel{\delta}{\rightarrow} 2 \mathbb{Z}^{2} \stackrel{i_{0}}{\hookrightarrow} 2 \mathbb{Z}$ \\
\hline $\mathrm{CII}^{\mathcal{R}_{++}}$ & CII & $\mathrm{CII}^{2}$ & $0 \stackrel{i_{1}}{\hookrightarrow} 0 \stackrel{j_{1}}{\hookrightarrow} 0 \stackrel{\delta}{\rightarrow} \mathbb{Z}^{2} \stackrel{i_{0}}{\hookrightarrow} \mathbb{Z}$ \\
\hline $\mathrm{C}^{\mathcal{R}_{+}}$ & $\mathrm{C}$ & $\mathrm{C}^{2}$ & $0 \stackrel{i_{1}}{\hookrightarrow} 0 \stackrel{j_{1}}{\hookrightarrow} 0 \stackrel{\delta}{\rightarrow} 0 \stackrel{i_{0}}{\hookrightarrow} 0$ \\
\hline $\mathrm{CI}^{\mathcal{R}_{++}}$ & CI & $\mathrm{CI}^{2}$ & $\mathbb{Z}^{2} \stackrel{i_{1}}{\hookrightarrow} \mathbb{Z} \stackrel{j_{1}}{\hookrightarrow} 0 \stackrel{\delta}{\hookrightarrow} 0 \stackrel{i_{0}}{\hookrightarrow} 0$ \\
\hline $\mathrm{AI}^{\mathcal{R}_{-}}$ & AII & A & $0 \stackrel{i_{1}}{\hookrightarrow} 0 \stackrel{j_{1}}{\hookrightarrow} 0 \stackrel{\delta}{\rightarrow} \mathbb{Z} \stackrel{i_{0}}{\hookrightarrow} 2 \mathbb{Z}$ \\
\hline$\overline{\mathrm{BDI}^{\mathcal{R}_{--}}}$ & CII & AIII & $\mathbb{Z} \stackrel{i_{1}}{\hookrightarrow} 0 \stackrel{j_{1}}{\hookrightarrow} 0 \stackrel{\delta}{\rightarrow} 0 \stackrel{i_{0}}{\hookrightarrow} 0$ \\
\hline $\mathrm{D}^{\mathcal{R}_{-}}$ & $\mathrm{C}$ & A & $0 \stackrel{i_{1}}{\hookrightarrow} 0 \stackrel{j_{1}}{\hookrightarrow} \mathbb{Z} \stackrel{\delta}{\rightarrow} \mathbb{Z} \stackrel{i_{0}}{\hookrightarrow} 0$ \\
\hline DIII $^{\mathcal{R}_{--}}$ & $\mathrm{CI}$ & AIII & $\mathbb{Z} \stackrel{i_{1}}{\hookrightarrow} \mathbb{Z} \stackrel{j_{1}}{\hookrightarrow} \mathbb{Z}_{2} \stackrel{\delta}{\rightarrow} 0 \stackrel{i_{0}}{\hookrightarrow} 0$ \\
\hline $\mathrm{AII}^{\mathcal{R}_{-}}$ & AI & A & $0 \stackrel{i_{1}}{\hookrightarrow} \mathbb{Z}_{2} \stackrel{j_{1}}{\hookrightarrow} \mathbb{Z}_{2} \stackrel{\delta}{\rightarrow} \mathbb{Z} \stackrel{i_{0}}{\hookrightarrow} \mathbb{Z}$ \\
\hline $\mathrm{CII}^{\mathcal{R}_{--}}$ & BDI & AIII & $\mathbb{Z} \stackrel{i_{1}}{\hookrightarrow} \mathbb{Z}_{2} \stackrel{j_{1}}{\hookrightarrow} 0 \stackrel{\delta}{\rightarrow} 0 \stackrel{i_{0}}{\hookrightarrow} \mathbb{Z}_{2}$ \\
\hline $\mathrm{C}^{\mathcal{R}_{-}}$ & $\mathrm{D}$ & A & $0 \stackrel{i_{1}}{\hookrightarrow} 0 \stackrel{j_{1}}{\hookrightarrow} 2 \mathbb{Z} \stackrel{\delta}{\rightarrow} \mathbb{Z} \stackrel{i_{0}}{\hookrightarrow} \mathbb{Z}_{2}$ \\
\hline $\mathrm{CI}^{\mathcal{R}_{--}}$ & DIII & AIII & $\mathbb{Z} \stackrel{i_{1}}{\hookrightarrow} 2 \mathbb{Z} \stackrel{j_{1}}{\hookrightarrow} 0 \stackrel{\delta}{\rightarrow} 0 \stackrel{i_{0}}{\hookrightarrow} 0$ \\
\hline $\mathrm{AI}^{\mathcal{C} \mathcal{R}_{-}}$ & $\mathrm{C}$ & CI & $\mathbb{Z} \stackrel{i_{1}}{\hookrightarrow} 0 \stackrel{j_{1}}{\hookrightarrow} 0 \stackrel{\delta}{\rightarrow} 0 \stackrel{i_{0}}{\hookrightarrow} 0$ \\
\hline $\mathrm{BDI}^{\mathcal{R}_{+-}}$ & $\mathrm{CI}$ & AI & $\mathbb{Z}_{2} \stackrel{i_{1}}{\hookrightarrow} \mathbb{Z} \stackrel{j_{1}}{\hookrightarrow} \mathbb{Z}^{2} \stackrel{\delta}{\rightarrow} \mathbb{Z} \stackrel{i_{0}}{\hookrightarrow} 0$ \\
\hline $\mathrm{D}^{\mathcal{C R}+}$ & AI & BDI & $\mathbb{Z}_{2} \stackrel{i_{1}}{\hookrightarrow} \mathbb{Z}_{2} \stackrel{j_{1}}{\hookrightarrow} \mathbb{Z}_{2}^{2} \stackrel{\delta}{\rightarrow} \mathbb{Z}_{2} \stackrel{i_{0}}{\hookrightarrow} \mathbb{Z}$ \\
\hline DIII $^{\mathcal{R}_{-+}}$ & BDI & $\mathrm{D}$ & $0 \stackrel{i_{1}}{\hookrightarrow} \mathbb{Z}_{2} \stackrel{j_{1}}{\hookrightarrow} \mathbb{Z}_{2}^{2} \stackrel{\delta}{\rightarrow} \mathbb{Z}_{2} \stackrel{i_{0}}{\hookrightarrow} \mathbb{Z}_{2}$ \\
\hline $\mathrm{AII}^{\mathcal{C} \mathcal{R}_{-}}$ & $\mathrm{D}$ & DIII & $2 \mathbb{Z} \stackrel{i_{1}}{\hookrightarrow} 0 \stackrel{j_{1}}{\hookrightarrow} 0 \stackrel{\delta}{\rightarrow} 0 \stackrel{i_{0}}{\hookrightarrow} \mathbb{Z}_{2}$ \\
\hline $\mathrm{CII}^{\mathcal{R}_{+-}}$ & DIII & AII & $0 \stackrel{i_{1}}{\hookrightarrow} 2 \mathbb{Z} \stackrel{j_{1}}{\hookrightarrow} 2 \mathbb{Z}^{2} \stackrel{\delta}{\rightarrow} 2 \mathbb{Z} \stackrel{i_{0}}{\hookrightarrow} 0$ \\
\hline $\mathrm{C}^{\mathcal{C R} \mathcal{R}_{+}}$ & AII & CII & $0 \stackrel{i_{1}}{\hookrightarrow} 0 \stackrel{j_{1}}{\hookrightarrow} 0 \stackrel{\delta}{\rightarrow} 0 \stackrel{i_{0}}{\hookrightarrow} 0$ \\
\hline $\mathrm{CI}^{\mathcal{R}_{-+}}$ & $\mathrm{CII}$ & $\mathrm{C}$ & $0 \stackrel{i_{1}}{\hookrightarrow} 0 \stackrel{j_{1}}{\hookrightarrow} 0 \stackrel{\delta}{\longrightarrow} 0 \stackrel{i_{0}}{\hookrightarrow} 0$ \\
\hline $\mathrm{AI}^{\mathcal{C} \mathcal{R}_{+}}$ & $\mathrm{D}$ & BDI & $\mathbb{Z}_{2} \stackrel{i_{1}}{\hookrightarrow} 0 \stackrel{j_{1}}{\hookrightarrow} 0 \stackrel{\delta}{\rightarrow} \mathbb{Z}_{2} \stackrel{i_{0}}{\hookrightarrow} \mathbb{Z}_{2}$ \\
\hline$\overline{\mathrm{BDI}^{\mathcal{R}_{-+}}}$ & DIII & $\mathrm{D}$ & $0 \stackrel{i_{1}}{\hookrightarrow} 2 \mathbb{Z} \stackrel{j_{1}}{\hookrightarrow} \mathbb{Z} \stackrel{\delta}{\rightarrow} \mathbb{Z}_{2} \stackrel{i_{0}}{\hookrightarrow} 0$ \\
\hline $\mathrm{D}^{\mathcal{C R} \mathcal{R}_{-}}$ & AII & DIII & $2 \mathbb{Z} \stackrel{i_{1}}{\hookrightarrow} 0 \stackrel{j_{1}}{\hookrightarrow} 0 \stackrel{\delta}{\rightarrow} 0 \stackrel{i_{0}}{\hookrightarrow} 2 \mathbb{Z}$ \\
\hline $\mathrm{DIII}^{\mathcal{R}_{+-}}$ & $\mathrm{CII}$ & AII & $0 \stackrel{i_{1}}{\hookrightarrow} 0 \stackrel{j_{1}}{\hookrightarrow} 2 \mathbb{Z} \stackrel{\delta}{\rightarrow} 2 \mathbb{Z} \stackrel{i_{0}}{\hookrightarrow} 0$ \\
\hline $\mathrm{AII}^{\mathcal{C} \mathcal{R}_{+}}$ & $\mathrm{C}$ & CII & $0 \stackrel{i_{1}}{\hookrightarrow} 0 \stackrel{j_{1}}{\longrightarrow} 0 \stackrel{\delta}{\rightarrow} 0 \stackrel{i_{0}}{\longrightarrow} 0$ \\
\hline $\mathrm{CII}^{\mathcal{R}_{-+}}$ & $\mathrm{CI}$ & $\mathrm{C}$ & $0 \stackrel{i_{1}}{\hookrightarrow} \mathbb{Z} \stackrel{j_{1}}{\hookrightarrow} \mathbb{Z} \stackrel{\delta}{\rightarrow} 0 \stackrel{i_{0}}{\hookrightarrow} 0$ \\
\hline $\mathrm{C}^{\mathcal{C R} \mathcal{R}_{-}}$ & AI & CI & $\mathbb{Z} \stackrel{i_{1}}{\hookrightarrow} \mathbb{Z}_{2} \stackrel{j_{1}}{\hookrightarrow} 0 \stackrel{\delta}{\rightarrow} 0 \stackrel{i_{0}}{\hookrightarrow} \mathbb{Z}$ \\
\hline $\mathrm{CI}^{\mathcal{R}_{+-}}$ & BDI & AI & $\mathbb{Z}_{2} \stackrel{i_{1}}{\hookrightarrow} \mathbb{Z}_{2} \stackrel{j_{1}}{\hookrightarrow} 2 \mathbb{Z} \stackrel{\delta}{\rightarrow} \mathbb{Z} \stackrel{i_{0}}{\hookrightarrow} \mathbb{Z}_{2}$ \\
\hline
\end{tabular}

are even under reflection. We (arbitrarily) take the condition that $H^{\prime}(0)$ be trivial to imply that $N^{\prime}(0)=0$.

In the language of Sec. IV, we thus see that $\mathcal{H}_{0}$ is the space of Hamiltonians in symmetry class AIII, whereas $\mathcal{M}_{0}$ is class A. Taking the zeroth and first fundamental groups of these two classes from Table I, we find that the corresponding exact sequence (35) reads

$$
0 \stackrel{i_{1}}{\hookrightarrow} \mathbb{Z} \stackrel{j_{1}}{\hookrightarrow} \mathbb{Z} \rtimes \mathbb{Z} \stackrel{\delta}{\rightarrow} \mathbb{Z} \stackrel{i_{0}}{\hookrightarrow} 0 .
$$

We conclude that $\pi_{1}\left(\mathcal{H}_{0}, \mathcal{M}_{0}\right)=\mathbb{Z} \rtimes \mathbb{Z}=\mathbb{Z}^{2}$.
The left and the right part of the exact sequence (C2) each contribute each a single topological index. We identify the first index $N^{\prime}$, which comes from the right part of the exact sequence, as the number $N^{\prime}(\pi)$ of even occupied states (under reflection symmetry) at the reflection symmetric point $k=\pi$. (Recall that by assumption $N^{\prime}(0)=0$.) The second topological index $W^{\prime}$ enumerates the equivalence classes of loops in class AIII, and can be calculated as twice the winding number of $\operatorname{det} r^{\prime}(k)$ between $k=0$ and $k=\pi$. [Note that $\operatorname{det} r^{\prime}(0)=1$, since $H^{\prime}(0)$ is trivial, whereas $\operatorname{det} r^{\prime}(\pi)= \pm 1$ and $\operatorname{det} r^{\prime}(k)=$ $\operatorname{det} r^{\prime}(-k)^{*}$.] Since an odd value of $W^{\prime}$ implies that odd parity of $N^{\prime}$ and vice versa, the allowed values of $\left(N^{\prime}, W^{\prime}\right)$ are subject to the constraint that $N^{\prime}+W^{\prime}$ be even.

Returning to the original Hamiltonian $H(k)=H(0) \oplus$ $H^{\prime}(k)$, we see that the addition of the $k$-independent part $H(0)$ does not affect the index $W^{\prime}$, whereas the first topological index has to be calculated as $N^{\prime}=N(\pi)-N(0)$, i.e., as the difference of the number of occupied even states at $k=\pi$ and $k=0$. To see that all allowed values for the two topological indices $N^{\prime}$ and $W^{\prime}$ are indeed attained, we construct generating Hamiltonians with $\left(N^{\prime}, W\right)=(1, \pm 1)$,

$$
H^{(1, \pm 1)}(k)=\cos k \sigma_{1} \mp \sin k \sigma_{2} \text {. }
$$

\section{The class $\mathrm{BDI}^{\mathcal{R}_{+-}}$}

At each $k$ point, the Hamiltonian satisfies $\mathcal{R} \mathcal{T}$ and $\mathcal{R} \mathcal{P}$ symmetries with $(\mathcal{R} \mathcal{T})^{2}=1,(\mathcal{R} \mathcal{P})^{2}=-1$, thus $\mathcal{H}_{0}$ is the space of Hamiltonians in symmetry class CI. Without loss of generality we take a representation in which $\mathcal{R} \mathcal{T}=K$, $\mathcal{R P}=\sigma_{2} K$, so that generic $H(k)$ can be written in the form

$$
H(k)=\left(\begin{array}{cc}
h(k) & d(k) \\
d(k) & -h(k)
\end{array}\right)
$$

with real and symmetric $h(k)$ and $d(k)$. Since $\mathcal{R}$ anticommutes with $\mathcal{P}$ and, hence, with $\mathcal{R} \mathcal{P}$, but commutes with $\mathcal{T}$ and $\mathcal{R} \mathcal{T}$, we may then use the unitary matrix $U_{\mathcal{R}}=\sigma_{3}$ to represent the reflection operation. This implies $d(k)=-d(-k)$ and, in particular, $d(0)=d(\pi)=0$, so that $\mathcal{M}_{0}$ is the space of class AI Hamiltonians. As in the previous subsection, we write $H(k)=H(0) \oplus H^{\prime}(k)$, where $H^{\prime}(0)$ is trivial, where we (again, arbitrarily) define "trivial" as all occupied states being odd under reflection. The exact sequence (35) then takes the form

$$
\mathbb{Z}_{2} \stackrel{i_{1}}{\hookrightarrow} \mathbb{Z} \stackrel{j_{1}}{\hookrightarrow} \mathbb{Z} \rtimes \mathbb{Z} \stackrel{\delta}{\rightarrow} \mathbb{Z} \stackrel{i_{0}}{\hookrightarrow} 0,
$$

leading to $\pi_{1}\left(\mathcal{H}_{0}, \mathcal{M}_{0}\right)=\mathbb{Z}^{2}$. We identify the two topological invariants $N^{\prime}$ and $W^{\prime}$ as the number of negative eigenvalues of $h^{\prime}(\pi)$ and as twice the winding number of $h(k)+i d(k)$ between $k=0$ and $k=\pi$, respectively. For the original Hamiltonian $H(k)$, the first invariant $N^{\prime}$ becomes the difference of the number of negative eigenvalues of $h(k)$ at $k=\pi$ and $k=0$. As in the previous case, the topological numbers are subject to the constraint that $N^{\prime}+W^{\prime}$ be even. The two generators are

$$
H^{(1, \pm 1)}(k)=\cos k \sigma_{3} \pm \sin k \sigma_{1} .
$$




\section{The class $\mathrm{CII}^{\mathcal{R}_{+-}}$}

Since $\mathcal{R}$ anticommutes with $\mathcal{P}$ and $\mathcal{P}^{2}=-1$ one has $(\mathcal{R P})^{2}=1$. Similarly, since $\mathcal{R}$ commutes with $\mathcal{T}$ and $\mathcal{T}^{2}=$ -1 one has $(\mathcal{R T})^{2}=-1$. This motivates the representation $\mathcal{R} \mathcal{T}=\tau_{2} \sigma_{3} K, \mathcal{R P}=\sigma_{2} K, \mathcal{R}=\sigma_{3}$. To get an explicit representation of matrices $H(k)$ for $0<k<\pi$, it is advantageous to define two alternative sets of Pauli matrices, $\mu_{1}=\sigma_{1}, \mu_{2}=$ $\tau_{2} \sigma_{3}, \mu_{3}=-\tau_{2} \sigma_{2}, \lambda_{1}=\tau_{1} \sigma_{1}, \lambda_{2}=\tau_{2}, \lambda_{3}=\tau_{3} \sigma_{1}$, so that $\mathcal{R P}=\mu_{1} K, \mathcal{R} \mathcal{T}=\mu_{2} K$. Using the $2 \times 2$ matrix structure corresponding to the $\mu$ matrices, we find that $H(k)$ for $0<k<\pi$ has the form

$$
H(k)=\left(\begin{array}{cc}
0 & r(k) \\
r(k)^{\dagger} & 0
\end{array}\right),
$$

with $r(k)$ complex antisymmetric and $r(k)=\lambda_{2} r^{*}(-k) \lambda_{2}$. We conclude that $\mathcal{H}_{0}$ is the space of gapped Hamiltonians in class DIII. To identify the symmetry of $H(k)$ at the symmetric momenta $k=0, \pi$ we use the original representation in terms of Pauli matrices $\sigma, \tau$, and find that $H(k)$ is of the form

$$
H(k)=\left(\begin{array}{cc}
h & 0 \\
0 & h^{*}
\end{array}\right), k=0, \pi,
$$

with $h=\tau_{2} h^{*} \tau_{2}$, which manifestly places $\mathcal{M}_{0}$ is in class AII. This gives the exact sequence

$$
0 \stackrel{i_{1}}{\hookrightarrow} 2 \mathbb{Z} \stackrel{j_{1}}{\hookrightarrow} 2 \mathbb{Z} \times 2 \mathbb{Z} \stackrel{\delta}{\rightarrow} 2 \mathbb{Z} \stackrel{i_{0}}{\hookrightarrow} 0 .
$$

The topological indices $N^{\prime}$ and $W^{\prime}$ are the difference in the number of occupied odd states at $k=0$ and $k=\pi$ (i.e., the difference of the number of negative eigenvalues of $h(k)$ for $k=0$ and $\pi$ ) and the winding number of $\operatorname{det} r(k)$ for $k$ between 0 and $\pi$, respectively. (Note that the condition $r(k)=\lambda_{2} r^{*}(-k) \lambda_{2}$ for $k=0, \pi$ implies that the Pfaffians of $r(0)$ and $r(\pi)$ are real, which implies that det $r(k)$ has an integer winding number for $k$ between 0 and $\pi$.) They are subject to the constraint that $N^{\prime}+W^{\prime}$ be even. Generators are

$$
H^{(1, \pm 1)}(k)=\tau_{2}\left(\sigma_{1} \cos k \pm \sin k \sigma_{3}\right) .
$$

\section{The class $\mathrm{D}^{\mathcal{C} \mathcal{R}_{+}}$}

The combined symmetry $\mathcal{P} \mathcal{C} \mathcal{R}$ serves as an effective timereversal symmetry at each $k$ point squaring to one, so that $\mathcal{H}_{0}$ is the class AI. At the reflection symmetric points, the $\mathcal{C} \mathcal{R}$ symmetry contributes as an additionally chiral symmetry that commutes with $\mathcal{P C} \mathcal{R}$, so that $\mathcal{M}_{0}$ is the class BDI. This gives the exact sequence

$$
\mathbb{Z}_{2} \stackrel{i_{1}}{\hookrightarrow} \mathbb{Z}_{2} \stackrel{j_{1}}{\hookrightarrow} \mathbb{Z}_{2} \times \mathbb{Z}_{2} \stackrel{\delta}{\rightarrow} \mathbb{Z}_{2} \stackrel{i_{0}}{\hookrightarrow} \mathbb{Z}
$$

To see that $\pi_{1}\left(\mathcal{H}_{0}, \mathcal{M}_{0}\right)=\mathbb{Z}_{2} \times \mathbb{Z}_{2}$ we first need to verify that the image of $i_{1}$ is the identity element in $\pi_{1}\left(\mathcal{H}_{0}\right)$. Here, we choose the representation $\mathcal{P C R}=K$ and $\mathcal{C R}=\tau_{3}$ and show that that the generator of $\pi_{1}\left(\mathcal{M}_{0}\right)$,

$$
H(k)=\tau_{1}\left(\sigma_{3} \cos k+\sigma_{1} \sin k\right),
$$

is mapped to the trivial element in $\pi_{1}\left(\mathcal{H}_{0}\right)$. This is indeed the case, since $H(k)$ is the diagonal sum of two nontrivial Hamiltonians in AI.

To find the group structure of $\pi_{1}\left(\mathcal{H}_{0}, \mathcal{M}_{0}\right)$ we need to inspect the topological indices $N^{\prime}$ and $W^{\prime}$ coming from the right and left parts of the exact sequence. We use the fact that at $k=0, \pi$ the Hamiltonian $H$ has the standard form

$$
H(k)=\left(\begin{array}{cc}
0 & o(k) \\
o(k)^{\mathrm{T}} & 0
\end{array}\right), k=0, \pi,
$$

where we take $o(k)$ to be orthogonal, $k=0, \pi$. (Orthogonal blocks $o(k)$ for $k=0, \pi$ can always be achieved by continuous deformation of $H(k)$.) This gives a reference basis for the occupied states given by the vectors

$$
\left|u_{\mathrm{ref}, j}(k)\right\rangle=\left(\begin{array}{c}
o_{j}(k) \\
-e_{j}
\end{array}\right), j=1,2, \ldots, N,
$$

where $o_{j}(k)$ is the $j$ th column of the orthogonal matrix $o(k), e_{j}$ is the $j$ th unit vector, and $2 N$ is the dimension of $H(k)$. We use $\left|u_{j}(k)\right\rangle$ to denote a basis for the occupied states, continuous as a function of $k$ and such that $\left|u_{j}(0)\right\rangle=\left|u_{\text {ref, }, j}(0)\right\rangle$. We can then define the topological indices $N^{\prime}=\operatorname{sign} \operatorname{det} o(0) \operatorname{det} o(\pi)$ and $W^{\prime}=\operatorname{sign} \operatorname{det}\left[\left\langle u_{i}(\pi) \mid u_{\mathrm{ref}, j}(\pi)\right\rangle\right]$. The corresponding generators are

$$
\begin{aligned}
& H^{(-1,1)}(k)=\tau_{1} \cos k+\tau_{3} \sin k, \\
& H^{(1,-1)}(k)=\tau_{1} \cos (2 k)+\tau_{3} \sin (2 k) .
\end{aligned}
$$

Note that $H^{(-1,1)}(k)$ has $\quad\left|u_{\mathrm{ref}}(0)\right\rangle=(1,-1) / \sqrt{2}$, $\left|u_{\mathrm{ref}}(\pi)\right\rangle=(-1,-1) / \sqrt{2}, \quad$ and $\quad|u(k)\rangle=(\cos (k / 2)-$ $\sin (k / 2),-\cos (k / 2)-\sin (k / 2)) / \sqrt{2}, \quad$ so that $W^{\prime}=1$. Similarly, $H^{(1,-1)}(k)$ has $\left|u_{\text {ref }}(0)\right\rangle=\left|u_{\text {ref }}(\pi)\right\rangle=(1,-1) / \sqrt{2}$, and $|u(k)\rangle=(\cos (k)-\sin (k),-\cos (k)-\sin (k)) / \sqrt{2}$, which gives $W^{\prime}=-1$. The $\mathbb{Z}_{2} \times \mathbb{Z}_{2}$ group structure follows upon verifying that the diagonal sum of each generator with itself has trivial indices.

\section{The class $D I I I^{\mathcal{R}_{-+}}$}

In this case $\mathcal{T} \mathcal{R}$ and $\mathcal{P} \mathcal{R}$ give effective time-reversal and particle-hole symmetries that both square to 1 . We may take the representation $\mathcal{T} \mathcal{R}=K, \mathcal{P} \mathcal{R}=\sigma_{3} K$, and $\mathcal{R}=\sigma_{2}$ and find that $H(k)$ is of the form

$$
H(k)=\left(\begin{array}{cc}
0 & r(k) \\
r(k)^{\mathrm{T}} & 0
\end{array}\right),
$$

with $r(k)$ real and $r(k)=-r(-k)^{\mathrm{T}}$. Hence we have $\mathcal{H}_{0}$ as class BDI and $\mathcal{M}_{0}$ as class D. The exact sequence (35) takes the form

$$
0 \stackrel{i_{1}}{\hookrightarrow} \mathbb{Z}_{2} \stackrel{j_{1}}{\hookrightarrow} \mathbb{Z}_{2} \times \mathbb{Z}_{2} \stackrel{\delta}{\rightarrow} \mathbb{Z}_{2} \stackrel{i_{0}}{\hookrightarrow} \mathbb{Z}_{2},
$$

so that $\pi_{1}\left(\mathcal{H}_{0}, \mathcal{M}_{0}\right)=\mathbb{Z}_{2} \times \mathbb{Z}_{2}$. To verify, we note that the image of $i_{0}$ is trivial, since $\operatorname{det} r(k)$ is always positive at $k=0$, $\pi$. The topological invariants are $N^{\prime}=\operatorname{sgn}[\operatorname{Pf} r(0) \operatorname{Pf} r(\pi)]$ and "winding number" $W^{\prime}$ of the real matrices $r(k)$ upon taking $k$ from 0 to $\pi$, which is the parity of the number twofold degenerate crossings of the (complex) eigenvalues of $r(k)$ on the negative real axis. The generators are of the from $(\mathrm{C} 16)$ with

$$
\begin{aligned}
& r^{(-1,1)}(k)=i \tau_{2} \cos k+\sin k, \\
& r^{(1,-1)}(k)=i \tau_{2} \cos (2 k)+\sin (2 k) .
\end{aligned}
$$


One verifies that the diagonal sum of each generator with itself gives a trivial element, so that the group structure is indeed $\mathbb{Z}_{2} \times \mathbb{Z}_{2}$.

\section{The class $\mathrm{BDI}^{\mathcal{R}_{-+}}$}

For this combination of symmetries we have $(\mathcal{R T})^{2}=-1$, $(\mathcal{R P})^{2}=1$, so that $\mathcal{H}_{0}$ belongs to the class DIII. We choose the explicit representations $\mathcal{R} \mathcal{T}=\sigma_{2} K, \mathcal{R} \mathcal{P}=K$, and $\mathcal{R}=\sigma_{3}$. The Hamiltonian $H(k)$ can then be cast into the form

$$
H(k)=i\left(\begin{array}{cc}
h(k) & d(k) \\
d(k) & -h(k)
\end{array}\right),
$$

where $h(k)$ and $d(k)$ are real and antisymmetric and $d(k)=$ $-d(-k)$. We see that $\mathcal{H}_{0}$ is in class DIII, whereas $\mathcal{M}_{0}$ is in class D. We again write $H(k)=H(0) \oplus H^{\prime}(k)$ with $H^{\prime}(0)$ trivial, calling $H^{\prime}(0)$ "trivial" if the Pfaffian $\operatorname{Pf} h^{\prime}(0)$ is positive. The exact sequence (35) then reads

$$
0 \stackrel{i_{1}}{\hookrightarrow} 2 \mathbb{Z} \stackrel{j_{1}}{\hookrightarrow} \mathbb{Z} \stackrel{\delta}{\rightarrow} \mathbb{Z}_{2} \stackrel{i_{0}}{\hookrightarrow} 0
$$

To see that the exact sequence (C20) indeed gives $\pi_{1}\left(\mathcal{H}_{0}, \mathcal{M}_{0}\right)=\mathbb{Z}$, we identify the topological indices $N^{\prime}$ and $W^{\prime}$ coming from the right and left parts of the exact sequence. In the present case, the index $N^{\prime}=\operatorname{Pf} h(\pi)$, which is a $\mathbb{Z}_{2}$ index, not an integer (as in the previous two examples). The index $W^{\prime}$ is twice the winding number of the Pfaffian $\operatorname{Pf}[h(k)+i d(k)]$ for $k$ between 0 and $\pi$. As in the previous two examples, we have the constraint that $N^{\prime}+W^{\prime}$ be even. Since $N^{\prime}$ is a $\mathbb{Z}_{2}$ index, this implies that the integer $W^{\prime}$ alone is sufficient to determine the topological classification of $H^{\prime}(k)$. Since $H(k)$ and $H^{\prime}(k)$ have the same winding numbers, the same topological index applies to the full Hamiltonian $H(k)$. As a generator we can take the four-band Hamiltonian

$$
H(k)=\left(\begin{array}{cc}
\tau_{2} \cos k & \tau_{2} \sin k \\
\tau_{2} \sin k & -\tau_{2} \cos k
\end{array}\right) .
$$

\section{The class DIII $^{\mathcal{R}--}$}

Here we choose a representation $\mathcal{T}=\tau_{2} K, \mathcal{P}=\tau_{2} \sigma_{2} K$, and $\mathcal{R}=\tau_{2}$, for which $H(k)$ is of the form (C4) with real and symmetric matrices $h(k)$ and $d(k)$ satisfying $h(k)=$ $\tau_{2} h(-k) \tau_{2}, d(k)=\tau_{2} d(-k) \tau_{2}$. The commutation with $\tau_{2}$ induces a two-dimensional representation of the complex numbers for $k=0, \pi$ [see the discussion following Eq. (37)], so that we conclude that $\mathcal{H}_{0}$ and $\mathcal{M}_{0}$ are classes CI and AIII, respectively. The exact sequence (35) then reads

$$
\mathbb{Z} \stackrel{i_{1}}{\hookrightarrow} \mathbb{Z} \stackrel{j_{1}}{\hookrightarrow} \mathbb{Z}_{2} \stackrel{\delta}{\rightarrow} 0 \stackrel{i_{0}}{\hookrightarrow} 0
$$

To show that $\pi_{1}\left(\mathcal{H}_{0}, \mathcal{M}_{0}\right)=\mathbb{Z}_{2}$, we need to show that the image of $i_{1}$ is $2 \mathbb{Z}$. To this end it is sufficient to consider the Hamiltonian

$$
H(k)=\sigma_{3} \cos k+\sigma_{1} \sin k,
$$

which is a generator for the loop space of AIII. Upon inclusion the loop space of CI, this Hamiltonian is mapped to $i_{1}[H(k)]=$ $H(k) \otimes \tau_{0}$, which is not the generator of the CI loop space. Instead, the generator of the CI loop space is $H(k) \oplus \sigma_{3}$, so that we conclude that the topological index of $i_{1}[H(k)]$ is equal to 2. The generator of the CI loop space is also the generator of $\pi_{1}\left(\mathcal{H}_{0}, \mathcal{M}_{0}\right)$.

\section{The class $\mathrm{C}^{\mathcal{C} \mathcal{R}_{-}}$}

For this symmetry class $\mathcal{H}_{0}$ is class AI, whereas $\mathcal{M}_{0}$ is class CI. We need to prove that the image of $i_{1}$ is whole of $\mathbb{Z}_{2}$. To this end, it is enough to verify that the particular example

$$
H(k)=\sigma_{3} \cos k+\sigma_{1} \sin k,
$$

with symmetries $\mathcal{T}=K$ and $\mathcal{P}=\sigma_{2} K$, is an element of $\mathrm{CI}$ and is mapped to a nontrivial loop in AI.

\section{The class $\mathrm{CII}^{\mathcal{R}--}$}

Here one has $\mathcal{H}_{0}$ is BDI and $\mathcal{M}_{0}$ is AIII. Similarly to the previous case, we need to show that the image of $i_{1}$ is $\mathbb{Z}_{2}$. Again, it is sufficient to find an example,

$$
H(k)=\sigma_{1} \cos k+\sigma_{2} \sin k, 0 \leqslant k \leqslant 2 \pi,
$$

with chiral symmetry given by $\mathcal{C}=\sigma_{3}$. One verifies that this example constitutes a loop in AIII. In order to identify the above Hamiltonian as a loop in BDI, one has to use the twodimensional representation of the complex numbers, see the discussion following Eq. (37), which turns $H(k)$ into a matrix of the form $(\mathrm{C} 1)$ with

$$
r(k)=\cos k-i \tau_{2} \sin k,
$$

which is a nontrivial loop in BDI.

\section{The class $\mathrm{CI}^{\mathcal{R}_{--}}$}

For this symmetry class, we have $(\mathcal{R} \mathcal{P})^{2}=1,(\mathcal{R} \mathcal{T})^{2}=$ -1 , which motivates the representation $\mathcal{R} \mathcal{P}=\sigma_{1} K, \mathcal{R} \mathcal{T}=$ $\tau_{3} \sigma_{2} K$, and $\mathcal{R}=\sigma_{3}$. This implies that $\mathcal{H}_{0}$ is class DIII. To get an explicit representation of matrices $H(k)$ for $0<k<\pi$, it is advantageous to define two alternative sets of Pauli matrices $\mu_{1}=\sigma_{1}, \mu_{2}=\tau_{3} \sigma_{2}, \mu_{3}=\tau_{3} \sigma_{3}, \lambda_{1}=\tau_{1} \sigma_{1}, \lambda_{2}=$ $\tau_{2} \sigma_{1}, \lambda_{3}=\tau_{3}$, so that $\mathcal{R P}=\mu_{1} K, \mathcal{R} \mathcal{T}=\mu_{2} K$. Using the $2 \times 2$ matrix structure corresponding to the $\mu$ matrices, we find that $H(k)$ for $0<k<\pi$ has the form (C7) with $r(k)$ complex antisymmetric. For $k=0, \pi$, it is more convenient to use the original Pauli matrices $\sigma, \tau$ and one finds that $H$ has the form

$$
H(k)=\left(\begin{array}{cc}
h & 0 \\
0 & \tau_{3} h^{*} \tau_{3}
\end{array}\right), k=0, \pi,
$$

where the Hermitian $h$ satisfies the additional constraint $h=$ $-\tau_{3} h \tau_{3}$. This places $H(0)$ and $H(\pi)$ in class AIII.

In order to resolve the exact sequence, we have to show that the image of $i_{1}$ is all of $2 \mathbb{Z}$. Here, we consider the example

$$
h(k)=\tau_{1} \cos k+\tau_{2} \sin k, 0 \leqslant k \leqslant 2 \pi,
$$

which is a nontrivial "loop" in AIII. Seen as a loop in DIII, one has $r(k)=\lambda_{2} \sin k+i \lambda_{2} \cos k$, which indeed is the generator of the first homotopy group.

\section{The class $\mathrm{CI}^{\mathcal{R}_{+-}}$}

We have $(\mathcal{R P})^{2}=(\mathcal{R} \mathcal{T})^{2}=1$, which motivates the representation $\mathcal{R} \mathcal{T}=K, \mathcal{R} \mathcal{P}=\sigma_{3} K, \mathcal{R}=\sigma_{1}$, from which it 
follows that $H(k)$ is of the form (C7) with $r(k)$ real and $r(k)=r(-k)^{\mathrm{T}}$. It follows that $\mathcal{H}_{0}$ is class BDI and $\mathcal{M}_{0}$ is class AI. We have to show that $i_{1}$ is surjective. Here, we consider the example

$$
r(k)=\tau_{3} \cos k+\tau_{1} \sin k, 0 \leqslant k \leqslant 2 \pi,
$$

which is nontrivial when seen as a loop in $\mathcal{H}_{0}$.

\section{APPENDIX D: INCREASING THE UNIT CELL SIZE IN CLASS AII $^{\mathcal{R}-}$ BY AN ODD NUMBER}

In this section, we show how to increase the size of the unit call for a Hamiltonian $H(k)$ with anticommuting $\mathcal{R}$ and $\mathcal{R} \mathcal{T}=K$ symmetries by a factor $2 n+1$, and show that this procedure does not change the topological invariant (39).

We define the Hamiltonian $\tilde{H}(k)$ on the smaller Brillouin zone $k \in[-\pi /(2 n+1), \pi /(2 n+1)]$ as

$$
\tilde{H}(k)=R^{T}(k)\left[\sum_{\substack{\oplus \\ l=-n}}^{n} H\left(k+\frac{2 l \pi}{2 n+1}\right)\right] R(k),
$$

where the orthogonal matrix $R(k)$ is introduced to enforce the $2 \pi /(2 n+1)$ periodicity of the folded Hamiltonian $\tilde{H}(k)$ while keeping $\tilde{H}(k)$ real,

$$
\begin{aligned}
R(k) & =\sum_{l=-n}^{n} e^{i n l k}\left|v_{l}\right\rangle\left\langle v_{l}\right|, \\
\left|v_{l}\right\rangle & =\sum_{m=-n}^{n} e^{i m l k}\left|e_{m}\right\rangle,
\end{aligned}
$$

$\left|v_{l}\right\rangle$ are eigenvectors of a circular permutation. The above Hamiltonian satisfies same symmetry relations as $H(k)$ with $\mathcal{R} \mathcal{T}=K$ and $\tilde{\mathcal{R}}=O \otimes \mathcal{R}$ with $O_{i j}=\delta_{i,-j}$.

The folded Hamiltonian has the negative-eigenvalue eigenvectors

$$
\left|\psi_{l a}\right\rangle=R(-k)\left|e_{l}\right\rangle \otimes\left|u_{a}^{-}\left(k+\frac{2 \pi l}{2 n+1}\right)\right\rangle,
$$

where $\left|u_{a}^{-}(k)\right\rangle$ are Bloch wave functions with negative energies. The matrix $\tilde{w}_{i j, a b}$ from Eq. (39) for the Hamiltonian $\tilde{H}(k)$ reads

$$
\begin{aligned}
\tilde{w}_{i j, a b}(k)= & \left\langle\psi_{i a}(-k)|\tilde{\mathcal{R}}| \psi_{j a}(k)\right\rangle^{*} \\
= & {[R(k) O R(-k)]_{i j} w_{a b}\left(k+\frac{2 \pi j}{2 n+1}\right) } \\
= & {[R(k) O R(-k)]_{i j} } \\
& \times\left\langle u_{a}^{-}\left(-k+\frac{2 \pi i}{2 n+1}\right)|\mathcal{R}| u_{b}^{-}\left(k+\frac{2 \pi j}{2 n+1}\right)\right\rangle .
\end{aligned}
$$

Since $w_{a b}(k)$ is an orthogonal matrix we have det $w_{a b}(k)=$ 1. For $k=0, \pi /(2 n+1)$, the block on the diagonal is $w_{a b}(0)$ and $w_{a b}(\pi)$, respectively, thus $\operatorname{Pf}[\tilde{w}(0)]=\operatorname{Pf}[w(0)]$ and $\operatorname{Pf}\left[\tilde{w}\left(\frac{\pi}{2 n+1}\right)\right]=\operatorname{Pf}[w(\pi)]$.

\section{APPENDIX E: NUMERICAL SIMULATIONS OF SECOND DESCENDANT $\mathbb{Z}_{2}$ PHASE}

In this section, we show how to carry out the analysis of Sec. V numerically for a concrete example. We consider a $2 \mathrm{~d}$ model with eight-band Hamiltonian in class $\mathrm{CII}^{\mathcal{R}_{--}}$for which it was previously argued that it exhibits a subtle instability in the presence of disorder [49]. After performing Fourier transform to real space, assuming the lattice to be square, the Hamiltonian reads

$$
\begin{aligned}
H= & \sum_{x_{i}, x_{j}, y} h\left(k_{x}, x_{i}, x_{j}, y\right)\left|x_{i}, y\right\rangle\left\langle x_{j}, y\right| \\
& +\frac{1}{2}\left(\tau_{3}+i \mu_{1} \tau_{1} \sigma_{2}\right)\left|x_{i}, y\right\rangle\left\langle x_{i}, y+1\right|+\text { H.c. }
\end{aligned}
$$

with the matrix elements given by

$$
\begin{aligned}
h\left(x_{i}, x_{j}\right)= & {\left[m \tau_{3}+\Delta(|x|) \tau_{2} \sigma_{2}\right] \delta_{x_{i}, x_{j}}+\frac{1}{2}\left(\tau_{3}+i \tau_{1} \sigma_{1}\right) \delta_{x_{i}, x_{j}-1} } \\
& +\frac{1}{2}\left(\tau_{3}-i \tau_{1} \sigma_{1}\right) \delta_{x_{i}, x_{j}+1}
\end{aligned}
$$

with the symmetries defined as $\mathcal{T}=\sigma_{2} K, \mathcal{P}=\mu_{2} \tau_{1} K$, and $\mathcal{R}=\sigma_{2}$. For numerical simulations, we assume the unit-cell size to be $M$ and express the above Hamiltonian in the following basis states,

$$
\left|k_{x}, \tilde{x}_{i}, y\right\rangle=\sqrt{\frac{M}{2 \pi}} \sum_{m=-\infty}^{\infty} e^{-i k_{x} m M}\left|\tilde{x}_{i}+m M, y\right\rangle,
$$

which are $2 \eta$-periodic functions of $k_{x}$ with $\eta=\pi / M$. We assume $\Delta(x)$ to be a spatially noncorrelated Gaussian variable, with mean $\bar{\Delta}$ and standard deviation $\delta$. Additionally, we take the origin of the coordinate system to be on the reflection plane, which assures that the $\Delta(|x|)$ term is reflection symmetric. A nonzero value of $\bar{\Delta}$ shifts the position of the two Dirac cones away from the mirror line [49]. Each lattice site depicted on Fig. 4 contains eight orbitals defining the space on which the Pauli matrices $\mu_{i}, \tau_{i}$, and $\sigma_{i}$ act. Physically, $\mu_{i}, \tau_{i}$, and $\sigma_{i}$ can be treated as particle-hole, one-half spin, and pseudospin degrees of freedom, respectively [49]. The above Hamiltonian is in the topologically nontrivial phase for $|m|<1+\sqrt{1-\bar{\Delta}^{2}}$.

We first numerically calculate the topological invariant defined by Eq. (39). To this end, we consider the setup depicted in Fig. 4, where two leads with a finite width are attached to the system. For the Hamiltonian of the leads, we take

$$
H_{L}=\sum_{x, y}\left(m \tau_{3}|x, y\rangle\left\langle x, y\left|+\frac{1}{2} \tau_{3}\right| x, y\right\rangle\langle x+1, y|+\text { H.c. }\right) \text {, }
$$

which also belongs to the class $\mathrm{CII}^{\mathcal{R}_{--}}$. For the above choice of the Hamiltonian, the number of propagating modes at $\varepsilon=0$ is equal to the total number of orbitals within the unit cell. We use software package KWANT [64] to numerically calculate the reflection matrix from the upper lead at $\varepsilon=0$. Next, using Eq. (12), we obtain the 1d Hamiltonian $H\left(k_{x}\right)$ in class $\mathrm{AII}^{\mathcal{R}_{-}}$with time-reversal symmetry given by Eq. (14). After applying a unitary transformation to the basis where $H\left(k_{x}\right)$ is real, we perform an exact diagonalization for each $k_{x} \in[0, \pi]$. In order to apply Eq. (39), we need a continuous basis of the negative energy manifold, $\left|u_{a}^{-}\left(k_{x}\right)\right\rangle$, which we find by projecting $\left|u_{a}^{-}\left(k_{x}-\delta k_{x}\right)\right\rangle$ onto the negative energy space at $k_{x}$ 


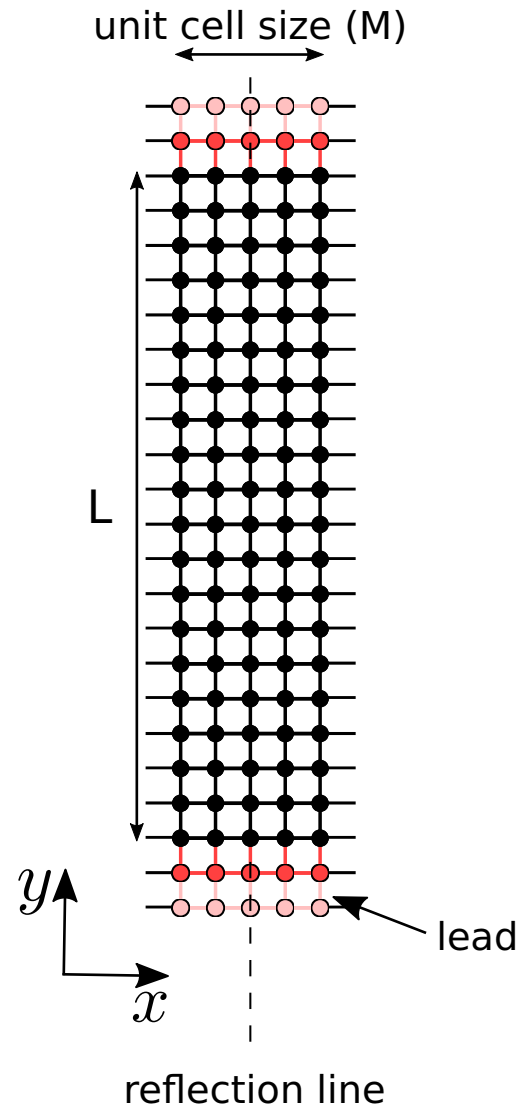

FIG. 4. Schematic illustration of the transport setup we consider. We consider two leads (red) that run along the $y$ direction, attached to the system (black). Twisted periodic boundary conditions (corresponding to the wave number $k_{x}$ ) are applied in the $x$ direction, with unit-cell size $M$. The whole system (together with the leads) is reflection symmetric with reflection plane parallel to the $y$ axis. Each site (point) contains eight orbitals. The system size $L$ in the $y$ direction is chosen to be large enough that $r\left(k_{x}\right)$ is unitary.

followed by orthonormalization procedure, where $\delta k_{x}$ is the step size. Figure 5 shows that topological invariant remains unchanged when disorder is considered and unit-cell size is being varied, in accordance with analysis from Sec. V.

We next show how to numerically determine, from the reflection matrix, the presence of the edge states in the $x$ direction and compare these results with the exact diagonalization of the full two-dimensional Hamiltonian (E1). We show the results for the case of doubled unit cell $(M=2)$ but all the conclusions that follow are valid for arbitrary $M$. Figure 6(a) shows the band structure for $k_{x} \in[0, \pi]$. There are four Dirac cones, two at the upper edge and two at two at the lower edge. Since we take $\bar{\Delta} \neq 0$, the Dirac cones of each edge are moved away from $k_{x}=0$ line.

In order to apply Eq. (45) we consider leads that are infinite in the $x$ direction so that $k_{x}$ is conserved. For $r^{\prime}\left(k_{x}\right)$, we take a reflection matrix that transforms the incoming to the outgoing modes without affecting the mode wave function within the unit cell. The reflection matrix $r\left(k_{x}\right)$ we obtain numerically by considering scattering from the upper lead for each $k_{x} \in[0, \pi]$. Finally, we transform the unitary operator $r\left(k_{x}\right) r^{\prime}\left(k_{x}\right)$ to the basis that is $k_{x}$ independent and perform the

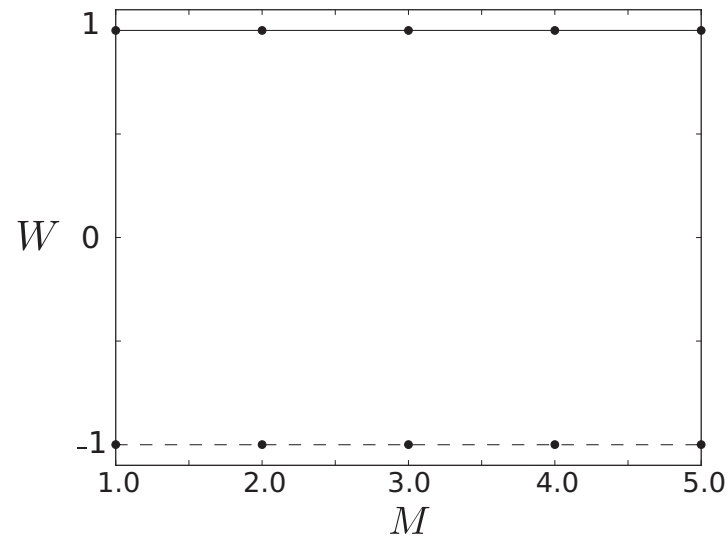

FIG. 5. The dependence of topological invariant (39) on the unitcell size $M$. The solid line is for $m=3$ (topologically trivial), whereas the dashed line is for $m=1$ (topologically nontrivial). The remaining parameters take the values $L=20, \bar{\Delta}=0.1$, and $\delta=0.03$.

exact diagonalization. Figure 6(b) shows the phases $\Phi$ of the obtained eigenvalues. By comparing Figs. 6(a) and 6(b), we conclude that the position of the Dirac cone (on the upper edge) and the appearance of the eigenvalue equal to one (i.e., $\Phi$ equal to zero) of the product $r\left(k_{x}\right) r^{\prime}\left(k_{x}\right)$ occur at the same $k_{x}$

(a)

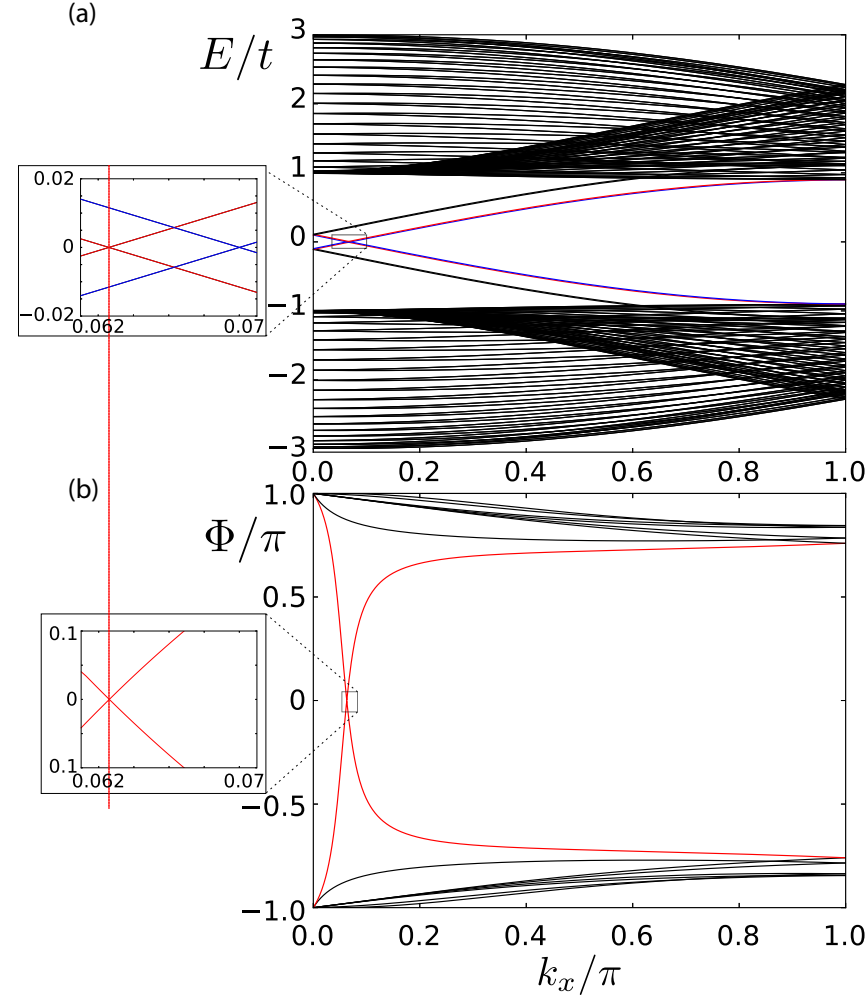

FIG. 6. (a) Dispersion over half of the Brillouin zone $k_{x} \in[0, \pi]$ for the system depicted in Fig. 4. The inset shows a closeup of the spectra that contains two Dirac-like dispersions, the red (blue) states being localized on the top (bottom) edge of the sample. (b) Phases $\Phi$ of the eigenvalues of the unitary operator $r\left(k_{x}\right) r^{\prime}\left(k_{x}\right)$, where $r\left(k_{x}\right)$ is the reflection matrix from the upper lead. The inset show that the position of the red Dirac cone matches the position where the phase is zero. The remaining parameters take the values $L=20, M=2$, $m=1, \bar{\Delta}=0.1$, and $\delta=0.03$. 


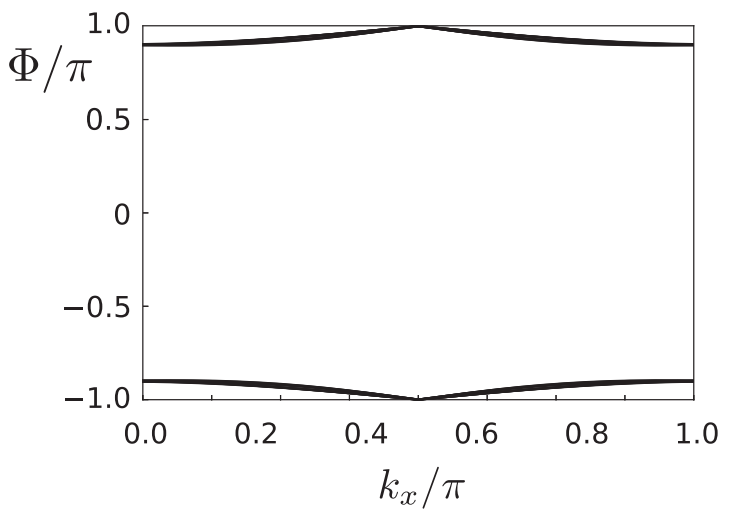

FIG. 7. Phases $\Phi$ of the eigenvalues of the unitary operator $r\left(k_{x}\right) r^{\prime}\left(k_{x}\right)$, where $r\left(k_{x}\right)$ is the reflection matrix from the system in the trivial phase. The remaining parameters take the values $L=20$, $M=2, m=3, \bar{\Delta}=0.1$, and $\delta=0.03$.

value. As explained in the main text, the presence of a single (or in general odd number) value of $k_{x}$ for which $\Phi=0$ is guaranteed by the topological invariant $W$ being nontrivial. For the sake of completeness, in Fig. 7 we show that there is no value of $k_{x}$ for which $\Phi=0$ [or an even number of such values, depending on the termination $\left.r^{\prime}\left(k_{x}\right)\right]$ if the topological invariant $W$ is trivial.

We also explicitly considered the perturbation which was previously argued to gap out the edge states the model (E1) (see Ref. [49]),

$$
\begin{aligned}
\delta h\left(x_{i}, x_{j}\right)= & -\frac{4 c}{\pi} \mu_{3} \tau_{2} \sigma_{1} \sin \left[\eta\left(x_{i}+x_{j}\right)\right] \\
& \times \frac{\cos \left[\delta_{1}\left(x_{i}-x_{j}\right)\right]}{x_{i}-x_{j}}
\end{aligned}
$$

where $\delta_{1}$ is a parameter. Its value should be chosen close to the position of an edge state Dirac cone. Although this perturbation breaks translation symmetry on the level of a single unit cell, the translation-symmetry breaking can be chosen to be commensurate with the underlying lattice by choosing rational $\eta=\pi / M$, so that it can be included in a Bloch Hamiltonian $H(k)$ for a unit cell size $M$. We confirmed that the perturbation (E5) opens a gap in the edge-state spectrum without closing the bulk gap for $c \gtrsim 1$. A gap in the edge-state spectrum is also opened up for $c \gtrsim 1$ if the perturbation is added near the sample edge only. However, the perturbation (E5) is not a local perturbation; it has long-range hopping, with a hopping amplitude decaying inversely proportional to distance. Hence, the corresponding Bloch Hamiltonian is not a continuous function of $k$, so that the topological classification, which assumed a continuous $k$ dependence, does not apply. To remain within the paradigm of the topological classification, we replace Eq. (E5) by a perturbation with a shortrange hopping term, while otherwise preserving the matrix structure,

$$
\begin{aligned}
\delta h\left(x_{i}, x_{j}\right)= & -\frac{2 c}{\pi} \mu_{3} \tau_{2} \sigma_{1} \sin \left[\eta\left(x_{i}+x_{j}\right)\right] \\
& \times \cos \left[\delta_{1}\left(x_{i}-x_{j}\right)\right] e^{-a \eta\left|x_{i}-x_{j}\right|} .
\end{aligned}
$$

This gives a continuous Bloch Hamiltonian $H(k)$. For this Hamiltonian, no gap in the edge-state spectrum was found for arbitrary strength of the perturbation if the perturbation is added near the sample edge only. If the perturbation is added uniformly in space, the opening of a gap in the surface state spectrum is preceded by the closing of the bulk gap, signaling a topological phase transition. We note that adding the local perturbation (E6) only at the edges can detach the spectra of the edge states from the bulk. Nevertheless, the edge states are topologically protected-the edge states' spectra and the wave functions are $4 \pi$ periodic with the property $E_{i}\left(k_{x}+2 \pi\right)=$ $-E_{i}\left(k_{x}\right)$, thus ensuring the presence of an edge state at zero energy. Such a Möbius twist of the spectra appears also in $4 \pi$-periodic Josephson junctions, [65] as well as in certain nonsymmorphic topological crystalline insulators [66-69].
[1] A. Altland and M. R. Zirnbauer, Phys. Rev. B 55, 1142 (1997).

[2] A. P. Schnyder, S. Ryu, A. Furusaki, and A. W. W. Ludwig, Phys. Rev. B 78, 195125 (2008).

[3] A. Kitaev, AIP Conf. Proc. 1134, 22 (2009).

[4] M. Stone, C.-K. Chiu, and A. Roy, J. Phys. A 44, 045001 (2011).

[5] X.-G. Wen, Phys. Rev. B 85, 085103 (2012).

[6] G. Abramovici and P. Kalugin, Int. J. Geom. Methods Mod. Phys. 09, 1250023 (2012).

[7] R. Kennedy and M. R. Zirnbauer, Commun. Math. Phys. 342, 909 (2016).

[8] R. Bott, Ann. Math. 70, 313 (1959).

[9] M. Atiyah, Quart. J. Math. Oxford Ser. 17, 367 (1966).

[10] M. Karoubi, in Handbook of K-Theory, edited by E. M. Friedlander and D. R. Grayson (Springer, Berlin, 2005).

[11] I. C. Fulga, F. Hassler, and A. R. Akhmerov, Phys. Rev. B 85, 165409 (2012).

[12] S. Ryu and Y. Hatsugai, Phys. Rev. Lett. 89, 077002 (2002).
[13] M. Sato, Phys. Rev. B 73, 214502 (2006).

[14] J. C. Y. Teo, L. Fu, and C. L. Kane, Phys. Rev. B 78, 045426 (2008).

[15] B. Béri, Phys. Rev. B 81, 134515 (2010).

[16] K. Yada, M. Sato, Y. Tanaka, and T. Yokoyama, Phys. Rev. B 83, 064505 (2011)

[17] M. Sato, Y. Tanaka, K. Yada, and T. Yokoyama, Phys. Rev. B 83, 224511 (2011).

[18] A. P. Schnyder and S. Ryu, Phys. Rev. B 84, 060504 (2011).

[19] Y. Hatsugai, New J. Phys. 12, 065004 (2010).

[20] T. L. Hughes, E. Prodan, and B. A. Bernevig, Phys. Rev. B 83, 245132 (2011).

[21] L. Fu and E. Berg, Phys. Rev. Lett. 105, 097001 (2010).

[22] I. C. Fulga, B. van Heck, J. M. Edge, and A. R. Akhmerov, Phys. Rev. B 89, 155424 (2014).

[23] L. Fu and C. L. Kane, Phys. Rev. Lett. 109, 246605 (2012).

[24] Z. Ringel, Y. E. Kraus, and A. Stern, Phys. Rev. B 86, 045102 (2012). 
[25] R. S. K. Mong, J. H. Bardarson, and J. E. Moore, Phys. Rev. Lett. 108, 076804 (2012).

[26] T. Morimoto and A. Furusaki, Phys. Rev. B 89, 035117 (2014).

[27] H. Obuse, S. Ryu, A. Furusaki, and C. Mudry, Phys. Rev. B 89, 155315 (2014).

[28] R. Jackiw and C. Rebbi, Phys. Rev. D 13, 3398 (1976).

[29] J. Goldstone and F. Wilczek, Phys. Rev. Lett. 47, 986 (1981).

[30] W. A. Benalcazar, B. A. Bernevig, and T. L. Hughes, Science 357, 61 (2017).

[31] Z. Song, Z. Fang, and C. Fang, arXiv:1708.02952 [condmat.mes-hall].

[32] F. Schindler, A. M. Cook, M. G. Vergniory, Z. Wang, S. S. P. Parkin, B. A. Bernevig, and T. Neupert, arXiv:1708.03636 [cond-mat.mes-hall].

[33] M. Lin and T. L. Hughes, arXiv:1708.08457 [cond-mat.meshall].

[34] J. Langbehn, Y. Peng, L. Trifunovic, F. von Oppen, and P. W. Brouwer, arXiv:1708.03640 [cond-mat.mes-hall].

[35] C. Fang and L. Fu, arXiv:1709.01929 [cond-mat.mes-hall].

[36] R.-J. Slager, A. Mesaros, V. Juricic, and J. Zaanen, Nat. Phys. 9, 98 (2013).

[37] J. Kruthoff, J. de Boer, J. van Wezel, C. L. Kane, and R.-J. Slager, arXiv:1612.02007 [cond-mat.mes-hall].

[38] C. Fang, M. J. Gilbert, and B. A. Bernevig, Phys. Rev. B 86, 115112 (2012).

[39] C. Fang, M. J. Gilbert, and B. A. Bernevig, Phys. Rev. B 87, 035119 (2013).

[40] X.-J. Liu, J. J. He, and K. T. Law, Phys. Rev. B 90, 235141 (2014).

[41] A. Alexandradinata, C. Fang, M. J. Gilbert, and B. A. Bernevig, Phys. Rev. Lett. 113, 116403 (2014).

[42] F. Zhang, C. L. Kane, and E. J. Mele, Phys. Rev. Lett. 111, 056403 (2013).

[43] X.-Y. Dong and C.-X. Liu, Phys. Rev. B 93, 045429 (2016).

[44] P. Jadaun, D. Xiao, Q. Niu, and S. K. Banerjee, Phys. Rev. B 88, 085110 (2013).

[45] J. C. Y. Teo and T. L. Hughes, Phys. Rev. Lett. 111, 047006 (2013).

[46] W. A. Benalcazar, J. C. Y. Teo, and T. L. Hughes, Phys. Rev. B 89, 224503 (2014).

[47] A. Lau, J. van den Brink, and C. Ortix, Phys. Rev. B 94, 165164 (2016).

[48] Y.-M. Lu and D.-H. Lee, arXiv:1403.5558.

[49] C.-K. Chiu, H. Yao, and S. Ryu, Phys. Rev. B 88, 075142 (2013).
[50] T. Morimoto and A. Furusaki, Phys. Rev. B 88, 125129 (2013).

[51] K. Shiozaki and M. Sato, Phys. Rev. B 90, 165114 (2014).

[52] T. H. Hsieh, H. Lin, J. Liu, W. Duan, A. Bansil, and L. Fu, Nat. Commun. 3, 982 (2012).

[53] Y. Tanaka, Z. Ren, T. Sato, K. Nakayama, S. Souma, T. Takahashi, K. Segawa, and Y. Ando, Nat. Phys. 8, 800 (2012).

[54] S.-Y. Xu, C. Liu, N. Alidoust, M. Neupane, D. Qian, I. Belopolski, J. Denlinger, Y. Wang, H. Lin, L. Wray et al., Nat. Commun. 3, 1192 (2012).

[55] J. C. Y. Teo and C. L. Kane, Phys. Rev. B 82, 115120 (2010).

[56] A. M. Turner, Y. Zhang, R. S. K. Mong, and A. Vishwanath, Phys. Rev. B 85, 165120 (2012).

[57] For a reflection-symmetric lattice, there are two inequivalent reflection planes separated by half a lattice spacing; see the discussion in Sec. V. The unit cell must be chosen consistent with the choice of the reflection plane to ensure that the unit cell is mapped into itself under reflection and hence $U_{\mathcal{R}}$ is $k$ independent. Different choices of the reflection plane potentially lead to different values of topological invariants [62]. The presence or absence of topologically protected boundary states depends, however, on a difference of topological invariants across the sample boundary and remains independent of the choice of the reflection plane.

[58] C.-K. Chiu, J. C. Y. Teo, A. P. Schnyder, and S. Ryu, Rev. Mod. Phys. 88, 035005 (2016).

[59] J. C. Budich and B. Trauzettel, Phys. Status Solidi RRL 7, 109 (2013).

[60] C. Nash and S. Sen, Topology and Geometry for Physicists (Academic Press, San Diego, CA, 1988).

[61] L. Fu and C. L. Kane, Phys. Rev. B 76, 045302 (2007).

[62] K. Shiozaki, M. Sato, and K. Gomi, Phys. Rev. B 95, 235425 (2017).

[63] S. Ryu, A. P. Schnyder, A. Furusaki, and A. W. W. Ludwig, New J. Phys. 12, 065010 (2010).

[64] C. W. Groth, M. Wimmer, A. R. Akhmerov, and X. Waintal, New J. Phys. 16, 063065 (2014).

[65] A. Y. Kitaev, Phys. Usp. 44, 131 (2001).

[66] K. Shiozaki, M. Sato, and K. Gomi, Phys. Rev. B 93, 195413 (2016).

[67] Z. Wang, A. Alexandradinata, R. J. Cava, and B. A. Bernevig, Nature (London) 532, 189 (2016).

[68] P.-Y. Chang, O. Erten, and P. Coleman, Nat. Phys. 13, 794 (2017).

[69] Y. Yanase and K. Shiozaki, Phys. Rev. B 95, 224514 (2017). 